This item was submitted to Loughborough's Research Repository by the author.

Items in Figshare are protected by copyright, with all rights reserved, unless otherwise indicated.

\title{
Development of a UHPLC-MS/MS (SRM) method for the quantitation of endogenous glucagon and dosed GLP-1 from human plasma
}

\section{PLEASE CITE THE PUBLISHED VERSION}

http://dx.doi.org/10.4155/bio-2017-0021

\section{PUBLISHER}

(c) 2017 Future Science Ltd

\section{VERSION}

AM (Accepted Manuscript)

\section{PUBLISHER STATEMENT}

This work is made available according to the conditions of the Creative Commons Attribution-NonCommercialNoDerivatives 4.0 International (CC BY-NC-ND 4.0) licence. Full details of this licence are available at: https://creativecommons.org/licenses/by-nc-nd/4.0/

\section{LICENCE}

CC BY-NC-ND 4.0

\section{REPOSITORY RECORD}

Howard, James W., Richard G. Kay, Ben Jones, Jaimini Cegla, Tricia Tan, Steve Bloom, and Colin Creaser. 2019. "Development of a UHPLC-MS/MS (SRM) Method for the Quantitation of Endogenous Glucagon and Dosed GLP-1 from Human Plasma”. figshare. https://hdl.handle.net/2134/26217. 
Development of a UHPLC-MS/MS (SRM) method for the quantitation of endogenous glucagon and dosed GLP-1 from human plasma James W Howard ${ }^{1,2 \dagger}$, Richard G Kay ${ }^{3}$, Ben Jones ${ }^{4}$, Jaimini Cegla ${ }^{4}$, Tricia $\operatorname{Tan}^{4}$, Steve Bloom $^{4}$ and Colin S Creaser ${ }^{2}$ 1 LGC Limited, Newmarket Road, Fordham, Cambridgeshire, CB7 5WW, UK

2 Centre for Analytical Science, Department of Chemistry, Loughborough University, Leicestershire, LE11 3TU, UK

3 University of Cambridge, Institute of Metabolic Science, Cambridgeshire CB2 OQQ, UK 4 Imperial College, Department of Investigative Medicine, Hammersmith Hospital Campus, Du Cane Road, London, W12 0NN, UK

† Author for correspondence. Tel: +44 (0) 1638720 500. Fax: +44 (0)1638 724200

Email: james.howard@lgcgroup.com

\section{Abstract}

Background: The performance of glucagon and GLP-1 immunoassays is often poor, but few sensitive LC-MS/MS methods exist as alternatives. Results: We established the first multiplexed LC-MS/MS method avoiding immunoenrichment for the quantitation of endogenous glucagon (LLOQ $15 \mathrm{pg} / \mathrm{mL}$ ) and dosed GLP-1 (LLOQ $25 \mathrm{pg} / \mathrm{mL}$ ) in human plasma. Specificity of endogenous glucagon quantitation was assured using a novel approach with a supercharging mobile phase additive to access a sensitive qualifier SRM. Endogenous glucagon concentrations were within the expected range, and showed good reproducibility after extended sample storage. A cross-validation against established immunoassays using physiological study samples demonstrated some similarities between methods. Conclusion: The LC-MS/MS method offers a viable alternative to immunoassays for quantitation of endogenous glucagon, dosed glucagon and/or dosed GLP-1.

Keywords: glucagon, GLP-1, endogenous, plasma, LC-MS/MS, immunoassay, crossvalidation, supercharging mobile phase additive, $m$-NBA 
31 Glucagon and glucagon-like peptide-1 (GLP-1) are peptide hormones encoded by the proglucagon gene, and are released from the gene product via tissue specific post translational processing (Figure 1).

\begin{tabular}{|c|c|c|c|c|c|c|c|c|c|}
\hline \multirow{3}{*}{$\begin{array}{c}\text { Gut/ } \\
\text { Brain } \\
\text { Position }\end{array}$} & \multicolumn{3}{|c|}{ Glicentin } & & & \multirow[t]{2}{*}{ GLP-1 (7-36) } & & \multirow[t]{2}{*}{ GLP-2 } & \multirow[b]{3}{*}{$\begin{array}{l}159 \\
160\end{array}$} \\
\hline & \multirow[b]{2}{*}{$1-32$} & \multicolumn{2}{|c|}{ Oxyntomodulin } & & & & & & \\
\hline & & $33-61$ & $\begin{array}{c}62- \\
69\end{array}$ & $\begin{array}{l}70- \\
71\end{array}$ & $\begin{array}{l}72- \\
77\end{array}$ & $78-107$ & $108-125$ & 126- 158 & \\
\hline Amino acid & $\begin{array}{c}\text { RSLQDTEEKSRS } \\
\text { FSASQADPLSDP } \\
\text { DQMNEDKR }\end{array}$ & $\begin{array}{l}\text { HSQGTFTS } \\
\text { DYSKYLDS } \\
\text { RRAQDFV } \\
\text { QWLMNT }\end{array}$ & $\begin{array}{l}\mathrm{KR} \\
\mathrm{NR} \\
\mathrm{NN} \\
\mathrm{IA}\end{array}$ & KR & $\begin{array}{l}\mathrm{HD} \\
\mathrm{EF} \\
\mathrm{ER}\end{array}$ & $\begin{array}{c}\text { HAEGTFTSDV } \\
\text { SSYLEGQAAKE } \\
\text { FIAWLVKGR }\end{array}$ & $\begin{array}{c}\text { GRRDFPE } \\
\text { EVAIVEEL } \\
\text { GRR }\end{array}$ & $\begin{array}{l}\text { HADGSFSDEM } \\
\text { NTILDNLAARD } \\
\text { FINWLIQTKITD }\end{array}$ & RK \\
\hline Pancreas & & Glucagon & & & & Major pro-g & cagon fra & sment & \\
\hline
\end{tabular}

Figure 1

Proglucagon sequence and major processing products in the in the gut/brain and pancreas

Glucagon is released from pancreatic $\alpha$ cells and is a counter regulatory hormone that responds to hypoglycemia and fasting by stimulating glycogenolysis and gluconeogenesis, as well as hepatic fatty acid $\beta$-oxidation and ketogenesis [1]. It is also a biomarker for diseases such as diabetes and neurendocrine tumours [2]. GLP-1 is released via secretion from intestinal $L$ cells, and has primary roles in enhancing the $\beta$-cell insulin response to eating, enhancing $\beta$-cell survival, inhibiting gastric emptying, inhibiting glucagon secretion, and suppressing appetite [1][3][4], as well as being of interest as a biomarker [5]. Pharmacological administrations of glucagon are known to increase energy expenditure [1], and therefore it is of interest along with GLP-1 for the development of obesity treatments [6][7].

To study the physiological role of glucagon and GLP-1, and exploit their use as biomarkers, precise and accurate methods for determining their plasma concentrations are required. These are also needed to determine their pharmacokinetics in studies where these are dosed [6][7]. Traditionally such peptides are quantified using immunoassays. However precision and accuracy can be poor, as can be the correlation between assays [8][9][10][11][12][13]. This is often attributed to the potential for antibodies to cross-react with similar compounds, including inactive degradation fragments and metabolites. Specificity is particularly challenging for GLP-1 assays due to the large number of isoforms present that may cross-react. GLP-1 1-37, 7-37, and 9-37 are produced from differential cleavage of the pro-glucagon precursor [11]. In humans these primarily exist in C-terminal amidated isoforms (GLP 1-36NH $\mathrm{NH}_{2}, 7-36 \mathrm{NH}_{2}$, and 9-36NH $\mathrm{NH}_{2}$ [4] [11][14]. GLP-1 7-36 $\mathrm{NH}_{2}$ is the biologically active form and is referred to by the unqualified GLP-1 nomenclature. Similarly, some glucagon 
immunoassays assays are known to cross-react with proglucagon products glicentin and oyntomodulin [9]. Glucagon metabolites, some of which have only been recently reported [15], may also cross-react.

These concerns cast doubt on the integrity of some of the data in the literature. Crossvalidations to help assess the performance of different kits and laboratories can be impractical, as kits can be expensive, resources may not be available for training, and assay specific equipment may be required. For example only one of seven glucagon immunoassays recently evaluated used a standard microplate reader [9]. In addition radioimmunoassays (RIA) necessitate additional health and safety precautions during set-up and require specialised disposal of radioisotopes.

LC-MS/MS based methodologies can help to overcome some of these challenges. For example, specificity can be improved by monitoring SRM transitions that incorporate the full length peptide and a related fragment ion, which will exclude many structurally similar compounds, and extraction methodologies and chromatographic separations can be tailored to the peptides of interest. Furthermore qualifier SRM transitions can be monitored, provided sufficiently sensitive transitions can be determined, to ensure results are consistent with those achieved from the quantitation SRM [16][17][18][19]. Inter-lab cross validations are also easier as methods can be transferred between LC-MS/MS systems with limited reoptimisation.

However the application of LC-MS/MS for glucagon and GLP-1 quantitation has been limited, primarily due to sensitivity challenges. Typical human plasma glucagon concentrations are in the region of $30 \mathrm{pg} / \mathrm{mL}$, which can increase by $30-100 \mathrm{pg} / \mathrm{mL}$ as a result of hypoglycemia, but decrease to $\sim 3-7 \mathrm{pg} / \mathrm{mL}$ during hyperglycaemia [9]. GLP-1 is typically present at lower plasma concentrations, at approx. $13 \mathrm{pg} / \mathrm{mL}$ for fed and $2 \mathrm{pg} / \mathrm{mL}$ for fasted subjects [20].

Despite these challenges, some sensitive LC-MS/MS methods have been reported. We described a method capable of routinely quantifying glucagon to $25 \mathrm{pg} / \mathrm{mL}$ using $400 \mu \mathrm{L}$ plasma [21]. Another group recently described a multiplexed LC-MS/MS method capable of quantifying endogenous glucagon to $2.7 \mathrm{pg} / \mathrm{mL}$ and GLP-1 to $2.6 \mathrm{pg} / \mathrm{mL}$ using $500 \mu \mathrm{L}$ plasma [20], which was a refinement of their previous method using $1000 \mu \mathrm{L}$ [22]. However these methods used immunoaffinity enrichment with magnetic beads coated with antianalyte antibodies, adding expense and complexity to the method, and the methods had 
92 sensitivity. Methods for GLP-1 quantitation avoiding immunochemistry have much higher 93 LLOQs, for example $66 \mathrm{pg} / \mathrm{mL}$ in a recent paper [23].

94 In this study we refined and expanded our previous method, to improve sensitivity, 95 robustness and throughput, and to add GLP-1 as a secondary analyte. The method was 96 qualified using fit-for-purpose criteria based on key experiments from FDA [24] and EMA [25] 97 bioanalytical validation guidelines. We also describe the novel use of a supercharging mobile 98 phase additive, meta nitrobenzyl alcohol ( $m$-NBA), to obtain a sensitive qualifier SRM 99 transition to ensure specificity of endogenous glucagon quantitation. The method was cross100 validated against two established immunoassays for each analyte using the same 101 physiological study sample set. 


\section{Chemicals and Materials}

105 Certified human glucagon (HSQGTFTSDYSKYLDSRRAQDFVQWLMNT) was obtained from 106 EDQM (Strasbourg, France). The analogue internal standard (IS) (des-thr ${ }^{7}$-glucagon) 107 (HSQGTFSDYSKYLDSRRAQDFVQWLMNT) and stable isotope labelled (SIL) internal 108 standard (HSQGT- $\left[{ }^{13} \mathrm{C}_{9} ;{ }^{15} \mathrm{~N}\right] \mathrm{F}-T S D Y S K Y L D S R R A Q D F V Q W-\left[{ }^{13} \mathrm{C}_{6} ;{ }^{15} \mathrm{~N}\right] \mathrm{L}-\mathrm{MNT}$ ) were obtained from Bachem (Bubendorf, Switzerland). GLP-1 (7-36) amide

110 (HAEGTFTSDVSSYLEGQAAKEFIAWLVKGR - NH2) was also obtained from Bachem.

111 Water was produced by a Triple Red water purifier (Buckinghamshire, U.K.). All chemicals 112 and solvents were HPLC or analytical reagent grade and purchased from either Fischer 113 Scientific (Loughborough, UK) or Sigma Aldrich (St Louis, MO USA).

\section{$114 \quad$ Preparation of Stock and Spiking Solutions}

115 Stock solutions of glucagon and glucagon internal standards ( $1 \mathrm{mg} / \mathrm{mL}$ ), and stocks of 116 GLP-1 $(0.1 \mathrm{mg} / \mathrm{mL})$, were prepared in borosilicate glass vials using surrogate matrix $[\mathrm{MeOH}$ : $117 \mathrm{H}_{2} \mathrm{O}$ : Formic acid (FA): Bovine serum albumin (BSA), (20/80/0.1/0.1, v/v/v/w); typically $118200 \mathrm{~mL} \mathrm{H}_{2} \mathrm{O}, 800 \mathrm{~mL} \mathrm{MeOH}, 1 \mathrm{~mL} F A$ and $1 \mathrm{~g} \mathrm{BSA}$ ]. Combined glucagon and GLP-1 working 119 solutions were prepared by dilution with this solvent to create nine calibration standard 120 spiking solutions (300, 500, 900, 2000, 6000, 12000, 20000, $3700040000 \mathrm{pg} / \mathrm{mL}$ ), and six 121 quality control spiking solutions (300, 500, 900, 1500, 4000, $35000 \mathrm{pg} / \mathrm{mL})$. A mixed internal 122 standard working solution (ISWS) containing both analog glucagon and SIL glucagon was 123 similarly prepared at $20 \mathrm{ng} / \mathrm{mL}$. The stock and working solutions were prepared to a volume 124 of $10 \mathrm{~mL}$ and were stored at $-20^{\circ} \mathrm{C}$ when not in use, as we previously demonstrated that 125 glucagon solutions were stable under these conditions [21].

\section{$126 \quad$ Method Development}

127 The previously reported method [21] was refined to increase sensitivity, robustness and throughput. Various changes to the extraction procedure and LC conditions were investigated, as summarised in Supplementary information Table 1. SRM transitions corresponding to the SIL glucagon IS and GLP-1 were identified and optimised. Mobile phases were modified with various proportions of the supercharging additive $m$-NBA (meta nitrobenzyl alcohol) and formic acid to attempt to improve sensitivity. Further information is presented in Supplementary Information Sections 1-5. The resulting extraction and LCMS/MS methods used for the qualification are detailed below. 
136 A surrogate matrix based approach was used for glucagon quantitation. Plasma QCs, to represent samples, were created by diluting spiking solutions 20 -fold into EDTA plasma to 200 (MED) and 1750 (HIGH) pg/mL, and the QC concentration adjusted for the endogenous concentration. These were extracted and analysed according to the qualified extraction and LC-MS/MS methods described below. Calibration standards, QC LLOQs (precision and accuracy batches only) and QC LOWs were then prepared by spiking $20 \mu \mathrm{L}$ of the appropriate spiking solution into the collection plate, along with $20 \mu \mathrm{L}$ of ISWS and $160 \mu \mathrm{L}$ surrogate matrix. Taking into account the 2-fold concentration experienced by plasma samples ( $400 \mu \mathrm{L}$ of plasma sample is reconstituted into $200 \mu \mathrm{L}$ of solvent) this gave final calibration levels of $15,25,45,100,300,600,1000,1850$, and $2000 \mathrm{pg} / \mathrm{mL}$, and final QC levels of 15 (QC LLOQ) and 45 (QC LOW) pg/mL.

Endogenous GLP-1 could not be detected, and therefore GLP-1 quantitation was performed as per an exogenous compound with calibrants and QCs prepared in the sample matrix (EDTA Plasma). Spiking solutions were diluted 20 -fold into plasma to produce calibrants at $25,45,100,300,600,1000,1850$ and $2000 \mathrm{pg} / \mathrm{mL}$ and QC concentrations at 25 (LLOQ), 75 (LOW), 200 (MED), and $1750(\mathrm{HIGH}) \mathrm{pg} / \mathrm{mL}$. These were extracted according to the qualified extraction method.

\section{Qualified Extraction Method}

Plasma sample (EDTA) $(400 \mu \mathrm{L})$ was placed into a $2 \mathrm{~mL} 96$ well plate and $20 \mu \mathrm{L}$ of ISWS was added to all non-blank samples. This step was performed on ice to ensure analyte stability [26]. The plate was vortex mixed, and samples precipitated using $1.1 \mathrm{~mL}$ of ACN: $\mathrm{H}_{2} \mathrm{O}: \mathrm{NH}_{3}(72: 25: 0.1, \mathrm{v} / \mathrm{v} / \mathrm{v})$, vortex mixed, sonicated for 5 minutes, and then centrifuged for 10 minutes at $2300 \times \mathrm{g} .1 .2 \mathrm{~mL}$ of supernatant was transferred using an automated liquid handling system (Quadra Tower, TomTec, Connecticut, USA) to a $2 \mathrm{~mL}$ plate and evaporated to dryness at $40^{\circ} \mathrm{C}$ under nitrogen (ca 90 minutes). Samples were reconstituted in $800 \mu \mathrm{L} 2 \% \mathrm{NH}_{3}$ (aq) and then vortex mixed, before being extracted using solid phase extraction (SPE) as per our previously reported method [21] A Bond Elut Plexa 96 round-well SPE plate (30 mg) was conditioned using $1 \mathrm{~mL} \mathrm{MeOH}$, then equilibrated with $1 \mathrm{~mL} \mathrm{H}_{2} \mathrm{O}$. The samples were loaded, washed with $1 \mathrm{~mL} 5 \% \mathrm{MeOH}$ (aq), eluted with $2 \times 225 \mu \mathrm{L}$ ACN: $\mathrm{H}_{2} \mathrm{O}: \mathrm{FA}(75: 25: 0.1, \mathrm{v} / \mathrm{v} / \mathrm{v})$ into a $1 \mathrm{~mL}$ Lo-bind plate, and then evaporated under nitrogen at $40^{\circ} \mathrm{C}$, before being reconstituted in $200 \mu \mathrm{L} 0.2 \%$ FA (aq). The plate was centrifuged for 10 minutes at $2300 \times \mathrm{g}$, and $40 \mu \mathrm{L}$ of sample injected on to the LC-MS/MS system for analysis, or the plate stored at $4^{\circ} \mathrm{C}$ awaiting injection. The entire process took 1 working day. 
171 The LC-MS/MS system consisted of a Waters Acquity UPLC system (Waters Corporation, 172 Massachusetts, USA) coupled to an AB SCIEX 5500 (Applied Biosystems / MDS SCIEX, 173 Ontario, Canada) with an electrospray ion source.

174 Glucagon was separated on a Waters Acquity UPLC BEH C18 $1.7 \mu \mathrm{m}(2.1$ x $100 \mathrm{~mm})$ 175 column maintained at $60{ }^{\circ} \mathrm{C}$. The mobile phase consisted of $(A) 0.2 \%$ FA in acetonitrile $176(\mathrm{ACN})$ and $(\mathrm{B}) \quad 0.2 \% \mathrm{FA}(\mathrm{aq})$. The gradient for glucagon elution was $22-32 \% \mathrm{~A}$ over 2 177 minutes, as in our previous method [21], followed by a gradient of $32-38 \%$ over 0.8 minutes for GLP-1 elution. The column was then cleaned with $95 \%$ A for 0.75 minutes then reequilibrated at the starting conditions for 0.05 minutes. The flow rate was $0.8 \mathrm{~mL} / \mathrm{min}$ and the total gradient time was 3.6 minutes per sample.

The mass spectrometer was operated in positive ion mode with an electrospray voltage of $5500 \mathrm{~V}$. The source temperature was $600^{\circ} \mathrm{C}$, the curtain, $\mathrm{N}_{2}$ collision, GS1, and GS2 gases were set to 40, 8, 60, 40 psi respectively. The Q1 and Q3 quadrupoles were both operated at unit resolution. Entrance potentials of $10 \mathrm{~V}$ and collision exit cell potentials of $13 \mathrm{~V}$ were used. MS periods were used to maximise dwell times, and therefore sensitivity. The selected reaction monitoring (SRM) transitions $697.5 \rightarrow 694.0,677.4 \rightarrow 673.8$ and $702.2 \rightarrow 698.8$ for glucagon, analogue glucagon IS, and SIL glucagon IS were monitored between $0-2$ minutes. These transitions were attributed to $\left[\mathrm{M}+5 \mathrm{H}^{+}\right]^{5+} \rightarrow\left[\mathrm{M}+5 \mathrm{H}^{+}-\mathrm{NH}_{3}\right]^{5+}$ in each case. Transitions used the optimal CE of 20,18, and 20 respectively and all used dwell times of 60 ms with declustering potential (DP) of 80 . The SRM transitions $660.6 \rightarrow 656.9$ and $660.6 \rightarrow 752.0$, which were attributed to $\left[\mathrm{M}+5 \mathrm{H}^{+}\right]^{5+} \rightarrow\left[\mathrm{M}+5 \mathrm{H}^{+}-\mathrm{NH}_{3}\right]^{5+}$ and $\left[\mathrm{M}+5 \mathrm{H}^{+}\right]^{5+} \rightarrow \mathrm{y}_{20}{ }^{3+}$ respectively, were monitored for GLP-1 between 2 - 3.6 minutes. These transitions used the optimal CE of 16 and 22, and DP of 100 and 110 respectively and all used dwell times of $100 \mathrm{~ms}$.

\section{Qualified LC-MS/MS Method (m-NBA mobile phases)}

The same LC-MS/MS systems were used as above. However the mobile phase consisted of (A) $0.01 \%$ (FA) in ACN with $0.05 \% m-N B A$ and (B) $0.01 \% \mathrm{FA}\left(95 / 5 \mathrm{H}_{2} \mathrm{O} / \mathrm{ACN}\right)$ with $0.05 \%$ $m-N B A$. The addition of $A C N$ to the aqueous phase enabled solubility of the $m$-NBA. The gradient for glucagon elution was 18-28\% A over 2 minutes, then $28-34 \%$ over for 0.8 minutes for GLP-1 elution, before being cleaned and re-equilibrated as above. MS settings were as above, unless stated differently below. The selected reaction monitoring (SRM) transitions $581.5 \rightarrow 578.5,564.7 \rightarrow 561.8$ and $585.5 \rightarrow 582.4$ for glucagon, analogue glucagon IS and SIL glucagon IS were monitored between $0-1.75$ minutes. These transitions were 
attributed to $\left[\mathrm{M}+6 \mathrm{H}^{+}\right]^{6+} \rightarrow\left[\mathrm{M}+6 \mathrm{H}^{+}-\mathrm{NH}_{3}\right]^{6+}$ in each case. Transitions used the optimal $\mathrm{CE}$ of $13,12,13$ respectively and all used DP of 80 and dwell times of $60 \mathrm{~ms}$. The SRM transitions $550.6 \rightarrow 601.6$ and $550.6 \rightarrow 639.3$ were monitored for GLP-1 between 1.75 - 3.6 minutes. These transitions were attributed to $\left[\mathrm{M}+6 \mathrm{H}^{+}\right]^{6+} \rightarrow \mathrm{y}_{16}{ }^{3+}$ and $\left[\mathrm{M}+6 \mathrm{H}^{+}\right]^{6+} \rightarrow \mathrm{y}_{17}{ }^{3+}$ respectively. Transitions used the optimal DP of 80 and 70 respectively and both used CE of 19 and dwell times of $100 \mathrm{~ms}$.

\section{Qualification Procedure}

The qualification experiments were based on key experiments described in the FDA [24] and EMA [25] bioanalytical guidelines. Analyst v 1.6.2 (Applied Biosystems / MDS SCIEX, Ontario, Canada) was used to process data and to construct calibration lines. Duplicate calibration standards were analysed within each batch, and calibration lines were constructed using peak area ratio-concentration plots withlinear regression and $1 / x^{2}$ weighting.

For glucagon our acceptance criteria required that the accuracy of $\geq 75 \%$ of standards in each batch was within $15 \%$ (20\% at the LLOQ) of the nominal concentration, with those outside this excluded from the calibration line.. For precision and accuracy batches QCs needed mean accuracy within $20 \%$, with precision (CV) $\leq 20 \%$ at each level. In other batches $\geq 2 / 3$ of the individual QCs needed accuracy within $20 \%$, with at least one QC at each level. For GLP-1 similar acceptance criteria was used, expect that a $25 \%$ criteria was used for precision and accuracy at all levels.

Precision and accuracy of the method was assessed by analysis of replicate $(n=6)$ QC samples at at least four different concentrations within a batch. Selectivity was determined by inspecting chromatograms from six independent plasma EDTA samples for the presence of potentially interfering peaks.

The effect of the presence of matrix to the response of the analyte and internal standard was determined from six independent plasma EDTA samples. These were extracted and post spiked at the $200 \mathrm{pg} / \mathrm{mL}$ level, and compared to the mean response from samples in surrogate matrix taking into account endogenous concentrations. In addition QCs were fortified at $200 \mathrm{pg} / \mathrm{mL}$ in six independent Plasma EDTA matrices (1 aliquot of each), and in lithium heparin plasma $(n=6)$.

Stability in matrix was determined by spiking a plasma sample at $200 \mathrm{pg} / \mathrm{mL}$ and incubating it at room temperature or ice for $4 \mathrm{hr} 50$ minutes before extraction ( $\mathrm{n}=6$ replicates), and compared to a sample immediately extracted. The ability to re-inject extracts was assessed 
at the $P \& A$ QC levels after 42 days storage at $4^{\circ} \mathrm{C}$. Analyte recovery was evaluated by comparing samples $(n=6)$ spiked with $200 \mathrm{pg} / \mathrm{mL}$ analyte, with extracts post spiked at this level to represent $100 \%$ recovery. The 10 -fold dilution of a QC HIGH sample (1750 $\mathrm{pg} / \mathrm{mL})(\mathrm{n}=6)$ with EDTA plasma was used to demonstrate whether low samples volumes could be analysed.

Collection of samples from volunteers to assess endogenous glucagon concentrations

Blood was collected from 11 male and 11 female healthy volunteers using glass plasma collection tubes ( $5 \mathrm{~mL}$, EDTA anticoagulant), obtained from BD (Oxford, UK). Tubes were placed on ice immediately after sample collection and then centrifuged at $2300 \mathrm{xg}$ for 10 minutes to obtain plasma, which was stored at $-80^{\circ} \mathrm{C}$. Plasma was collected at the start of the working day and volunteers were not asked to change their usual eating regime.

Glucagon concentrations were determined using the qualified method with formic acid phases. Concentrations were determined upon the first freeze-thaw of the aliquot, as multiple cycles have been shown to affect quantitation [21]. The ability to extrapolate below the $15 \mathrm{pg} / \mathrm{mL}$ for glucagon limit of quantitation was assessed by determining precision and accuracy of surrogate matrix QCs $(n=6)$ diluted to 10 and $7.5 \mathrm{pg} / \mathrm{mL}$ levels.

Samples containing endogenous levels of glucagon above the lower limit of quantitation (15 $\mathrm{pg} / \mathrm{mL}$ ) were reanalysed after storage for up to 249 days at $-80^{\circ} \mathrm{C}$ to assess incurred sample reproducibility (ISR). Similarly a plasma pool from one male and one female individual was created from stored samples and analysed immediately after pooling and after additional storage for 42 days at $-80^{\circ} \mathrm{C}$.

Finally, extracts from a selection of samples $(n=9)$, and corresponding calibration standards and QC samples were reanalysed with the qualified method using $m$-NBA mobile phases to access a sensitive qualifier SRM to assure the methods selectivity.

\section{Collection of physiological study samples}

A $20 \mathrm{~mL}$ blood sample was collected from ten fasting healthy volunteers (West London National Research Ethics Committee, ref. 11/LO/1782), one volunteer dosed with glucagon (15 pmol/kg/min iv) (London Central Ethics and Research Committee (13/LO/0925), and two volunteers dosed with GLP-1 (16 pmol/kg/min sc) (13/LO/1510). Immediately after collection, $2 \mathrm{ml}$ samples were decanted into various collection tube types: 1) K3 EDTA anticoagulant (ref 368860 - Becton Dickinson Vacutainer System, NJ, USA) with the addition of aprotinin

269 (Bayer, Newbury, UK; final concentration 250 kallikrein inhibitor units/ml) and a DPPIV inhibitor (lle-Pro-Ile, Sigma-Aldrich, Dorset, UK; final concentration $10 \mu \mathrm{g} / \mathrm{ml}$ ); 2) K3 EDTA anticoagulant with the addition of aprotinin; 3) K3 EDTA anticoagulant with the addition of a 
272 DPPIV inhibitor; 4) K3 EDTA anticoagulant alone; 5) lithium heparin (ref 367883 - Becton

273 Dickinson Vacutainer System, NJ, USA) with the addition of aprotinin and a DPPIV inhibitor;

274 6) lithium heparin with the addition of aprotinin; 7) lithium heparin with the addition of a

275 DPPIV inhibitor and 8) lithium heparin alone. Following collection, tubes were placed on ice

276 and centrifuged at $2300 \times \mathrm{g}$ for 10 minutes to obtain plasma, which was stored at $-80^{\circ} \mathrm{C}$. Two

277 further aliquots, collected in K3 EDTA tubes with aprotinin and DPPIV inhibitor, underwent 1

278 and 2 further freeze/thaw cycles, respectively.

\section{$279 \quad$ Analysis of physiological study samples}

280 Samples of the same stabiliser type and anticoagulant from three of the ten fasting healthy volunteers were pooled together. One aliquot was reserved for endogenous level determination and a second aliquot was spiked with $200 \mathrm{pg} / \mathrm{mL}$ analyte. Samples were analysed using the qualified LC-MS/MS method with formic acid mobile phases to assess the effect that a change of sample matrix had upon quantitation.

The majority of the samples from five of the remaining seven fasting healthy volunteers (endogenous glucagon samples) were analysed by the LC-MS/MS method and by two immunoassays (50/50, 50/50 and 45/50 for the LC-MS/MS, first, and second immunoassay respectively). The first immunoassay was a Homogenous Time Resolved Fluorescence (HTRF®) sandwich immunoassay (Cisbio, Codolet, France) (analytical sensitivity $=12.0$ $\mathrm{pg} / \mathrm{mL}$ ). The second was a Milliplex MAP (multi-analyte profiling) Human Metabolic Hormone Magnetic Bead Panel (Merck Millipore, Darmstadt, Germany) (minimum detectable concentration $(\mathrm{MinDC})=13.0 \mathrm{pg} / \mathrm{mL}$ ), which involved a sandwich immunoassay with fluorescence detection. Samples from the other two fasting healthy volunteers were only analysed using one method format, and are therefore not considered further. The same formats were also used to analyse samples from the volunteer dosed with glucagon. Samples from the two volunteers dosed with GLP-1 were similarly analysed using the LCMS/MS method, and by either a Millipore active GLP-1 ELISA (Lowest standard $=6.6$ $\mathrm{pg} / \mathrm{mL}$ ) or the Milliplex MAP $(\mathrm{MinDC}=1.2 \mathrm{pg} / \mathrm{mL})$ described above.

The various measures of immunoassay sensitivity quoted by the manufactures (i.e. analytical sensitivity/ MinDC/ lowest calibration point) were taken as equivalent to the lower limit of quantitation (LLOQ) measurement of the LC-MS/MS method for the purpose of this study. For all immunoassays, intra-assay $\mathrm{CVs}$ were $<10 \%$ across the working range. 
306 Results and Discussion

$307 \quad$ Method Development-Re-optimisation

308 Initial development focused on re-optimisation of the glucagon method described previously 309 [21] to attempt to improve sensitivity, robustness, and throughput for glucagon analysis. 310 GLP-1 was the secondary analyte for the assay, and was not introduced at this stage. A 311 summary of the re-optimisation is presented in Supplementary Information Table 1, and

312 further details are given in Supplementary Information Sections 1-5.

313 The protein precipitation solvent volume used to extract the $400 \mu \mathrm{L}$ plasma samples was 314 lowered from $3.2 \mathrm{~mL}$ to $1.1 \mathrm{~mL}$. Such low plasma: solvent ratios were not previously 315 investigated as they would usually lead to high matrix effects, however the subsequent use 316 of SPE as part of the 2D extraction procedure minimised these. This alteration allowed the 317 assay to be transferred from $5 \mathrm{~mL}$ tubes into 96 well plates, enabling the use of an 318 automated liquid handling system to transfer the protein precipitation supernatant further 319 reducing extraction time and increasing robustness. Additionally it reduced the supernatant 320 evaporation time to ca 90 minutes, halving the extraction time to 1 working day, which is 321 similar to the incubation period for some glucagon immunoassays [9].

322 Following SPE the reconstituted samples contained less insoluble material than observed 323 previously [21], and upon analysis an interference peak (1.6 $\mathrm{min}$ ) close to glucagon's 324 retention time (1.7) was eliminated (Supplementary Information Figure 2a vs b). This may 325 be due to the lower transfer of solid protein precipitate using the automated system than with 326 a manual transfer. The reduced matrix content associated with the modified method also 327 eliminated the need for a lengthy column clean, shortening the LC gradient time to 3.6 328 minutes and increasing analyte signal in the plasma extracts.

329 A SIL glucagon IS was acquired to investigate whether this improved performance over the 330 analogue IS used previously. Performance was similar under standard conditions, however 331 the SIL IS gave better performance when matrix effects were high, and it was therefore 332 selected to maximise robustness. The other parameters investigated did not result in an 333 improvement of the method. 
336 LC-MS analysis of GLP-1 showed that the $[\mathrm{M}+3 \mathrm{H}]^{3+},[\mathrm{M}+4 \mathrm{H}]^{4+}$ and $[\mathrm{M}+5 \mathrm{H}]^{5+}$ charge states $337(\mathrm{~m} / \mathrm{z}=1009.5,825.1$, and 660.0) were observed using formic acid based mobile phases. The 338 latter two being most intense, and of similar intensity to each other (Figure 2a). As with glucagon [21], very little fragmentation was observed at lower collision energies and once a critical energy was reached numerous low intensity products were observed. Following optimisation and evaluation of sensitivity and linearity, the $660.6 \rightarrow 656.9$ and $660.6 \rightarrow 752.0$

342 SRM transitions were found to be similarly optimal, with sufficient sensitivity obtained at $34325 \mathrm{pg} / \mathrm{mL}$ for extracted plasma samples. Previously, either the $825.4 \rightarrow 946.3$ transition alone [20][22], or the 825.2 $\rightarrow 946.4$ transition summed with $825.2 \rightarrow 643.2$ [23] were found optimal. Differences in the LC-MS/MS systems used, including the use of nano or microflow LC in the previous studies, as well as variations in the nature of samples used for evaluation, may help to explain the discrepancies.

\section{Method Development-Supercharging Mobile Phase Additives}

One of the challenges for sensitive LC-MS/MS peptide bioanalysis is that signal is split over multiple charge states. Supercharging mobile phase additives, for example $m$-NBA, are known to alter the charge state distribution favouring higher charge states [27]. This is thought to be due to an increase in surface tension of the droplets in the electrospray source, decreasing the radius prior to columbic explosion, and consequently leading to charge concentration [28]. Sensitivity increases may result if the charge state distribution is reduced, or if the signal is increased due to improved analyte ionisability, as we have demonstrated for one large peptide in plasma extract [27]. However for glucagon whilst we previously demonstrated alterations in the charge state distribution $(3,4,5+$ to $4,5,6+)$ and an improvement in analyte signal in extracts, no overall increase in sensitivity (signal to noise) resulted due to similar increases in background noise [27].

It was decided to re-investigate the use of $m$-NBA to improve glucagon sensitivity with the re-optimised extraction method and to more fully investigate the effect of altering the $m$-NBA and FA content of the mobile phases. Furthermore it was planned to use the $m$-NBA method to provide a sensitive qualifier SRM to ensure assay specificity as described later, so an optimal method was required. The effect on GLP-1 was investigated, which similarly to glucagon demonstrated a shift in charge state distribution (Figure 2b). 

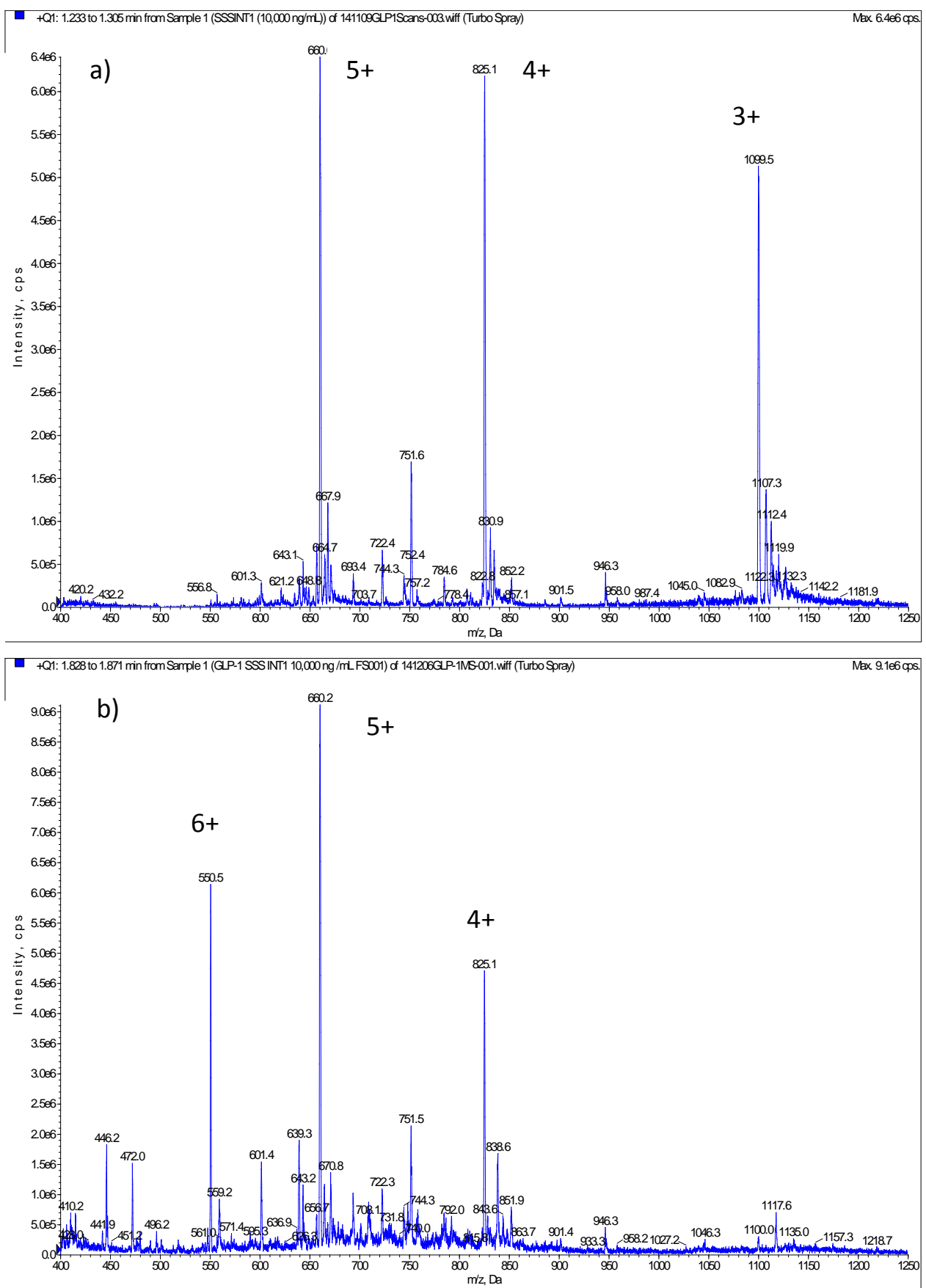

Figure 2- GLP-1 Full Scan MS Spectra- a) Formic acid mobile phases (Mobile $A=0.2 \%$ FA (ACN) Mobile $\mathrm{B}=0.2 \% \mathrm{FA}(\mathrm{aq}))$, b) Supercharging $(m-\mathrm{NBA})$ mobile phases (Mobile $\mathrm{A}=\mathrm{ACN}$ : $m$-NBA:FA $(100: 0.1: 0.1)$, Mobile $B=\mathrm{H}_{2} \mathrm{O}: A C N: F A: m-N B A(95: 5: 0.1: 0.1)$

373 The charge state distribution and signal intensity of glucagon and GLP-1 were affected by changes in the $m$-NBA and FA composition of the mobile phases in both solution and plasma extracts (Supplementary Information 5.1 - 5.3). Initial results suggested that higher levels of $m$-NBA favoured higher charge states (Supplementary Information 5.1.1) with the $5+$ and $6+$ charge state of glucagon being most dominant at $0.05 \%$ and $0.10 \% \mathrm{~m}$-NBA respectively. However further experiments suggested that $0.05 \% \mathrm{~m}$-NBA was optimal for both charge states (Supplementary Information 5.2). Significant changes in signal intensity 
occurred with the alteration of mobile phase formic acid content, which did not occur when using standard mobile phases (Supplementary Information 5.1 .2 vs 3.2). Interestingly mobile phases modified with low amounts of formic acid (e.g. $0.01 \%$ ) gave optimal signal for both analytes, regardless of whether they were in solution or plasma matrix, although the absence of acid led to very low signal demonstrating its necessity for efficient ionisation. Overall mobile phases modified with $0.05 \% \mathrm{~m}$-NBA $0.01 \%$ FA gave optimal signal in plasma and extract for both analytes.

Plasma extracts were analysed using both the optimal $m$-NBA and standard formic acid mobile phase methods (Supplementary Information 5.3). Similar sensitivity (signal-to-noise) was obtained using both conditions for glucagon and GLP-1, suggesting that $m$-NBA the method could provide a sensitive qualifier SRM. Method performance using $m$-NBA modified phases were further characterised during the establishment.

\section{Quantitation Strategy}

The endogenous nature of glucagon presents additional challenges as an authentic matrix free of analyte cannot be acquired, and therefore approaches such as standard addition, surrogate matrix or surrogate analyte must be considered as described previously [21]. This assay used the surrogate matrix based approach for glucagon quantitation, where calibration standards are prepared in an analyte free surrogate matrix, allowing low levels of glucagon in plasma samples to be quantified without extrapolation. Ideally surrogate calibrants and surrogate QC samples would be extracted alongside plasma samples, but this was not possible due to non-specific binding of the analyte in surrogate matrix to the extraction materials. Therefore non-extracted surrogate samples were used, which were added to the plate after plasma samples had been extracted.

Similarly, in such strategies it is common to evaluate the performance of plasma samples diluted with surrogate matrix to demonstrate parallelism between surrogate and authentic matrix [25][29][30]. However, this was not possible as dilution prevented the protein precipitation, which was required in the extraction procedure. Therefore the performance of QCs spiked with analyte on top of the endogenous levels against the surrogate calibration line was considered sufficient.

Acceptable sensitivity for is usually demonstrated by assessing whether the analyte response at the LLOQ level is at least 5 times the average response due to background noise [25]. It is then assumed that an unknown sample at the LLOQ concentration would also have a similarly acceptable response. However, this will not necessarily be the case for surrogate matrix assays, due to differences in the recovery and matrix suppression between 
415 the surrogate and authentic matrices. The previous method [21] had a mean analyte 416 recovery of $51.2 \%$ and matrix suppression (matrix factor $=0.746$ ), and therefore the signal-to417 noise $(\mathrm{S} / \mathrm{N})$ required at the LLOQ was 13.1 to ensure that $\mathrm{S} / \mathrm{N}$ for an authentic sample at the 418 LLOQ level $\geq 5$ (assuming an unchanged background level). However using the re-optimised 419 extraction method the IS response from surrogate matrix and plasma samples were similar 420 (Supplementary Information 5.4) demonstrating that recovery losses during plasma 421 extraction were compensated by matrix enhancement. The increase in the signal-to-noise 422 requirements of surrogate matrix LLOQs were therefore not required, and allowed establishment of a $15 \mathrm{pg} / \mathrm{mL}$ LLOQ with signal-to-noise $\geq 5$.

The assay was not sensitive enough to detect endogenous concentrations of GLP-1, therefore it was quantified using an exogenous quantitation strategy, where both calibrants and QCs were prepared in the authentic plasma matrix.

Qualification

The FDA [24] and EMA [25] bioanalytical validation guidelines do not formally consider large molecule or biomarker analysis by LC-MS/MS. However they are often used as the basis for such studies, as in this current work, as many experiments are applicable and alternative regulatory guidelines do not exist. When setting acceptance criteria for biomarker assays it is important to consider the natural variability of the biomarker, for glucagon plasma concentrations can vary widely $(3-130 \mathrm{pg} / \mathrm{mL})$, and therefore a very stringent acceptance criteria was not considered necessary. Considering this and the analytical challenges associated with the surrogate nature of the assay, a $20 \%$ value was used for glucagon precision and accuracy across all levels, which is often used for immunoassays. For GLP-1 a $25 \%$ criteria at all levels was used for the interpretation of data from the dosed subjects because of the analytical challenges presented by the lack or a SIL or closely related analogue IS.

\section{Glucagon}

The performance of the developed method was initially assessed using formic acid based mobile phases. The assay was linear over the $15-1000 \mathrm{pg} / \mathrm{mL}$ calibration range assessed. Signal to noise was $\geq 5$ at the LLOQ level (Figure 3a). Precision and accuracy (\%RE and $\%$ CV) were within the $20 \%$ acceptance criteria across all levels assessed (Supplementary Information Table 5).

447 Matrix enhancement was observed, however this was compensated by the enhancement of 448 the SIL IS, so it did not affect quantitation (Supplementary Information Table 6). The assay 
gave acceptable performance across 6 individuals matrices fortified with $200 \mathrm{pg} / \mathrm{mL}$ of 450 glucagon, and also in lithium heparin plasma providing further evidence that the assay was 451 not significantly affected a matrix changes (Supplementary Information Table 5). There were 452 no interferences in the selectivity samples near glucagon's retention time that could affect 453 the quantitation. Recovery, determined at $200 \mathrm{pg} / \mathrm{mL}$, was $50.7 \%$.

454 The performance of the method was similarly assessed using mobile phases modified with $455 m$-NBA, and comparable performance was observed (Supplementary Information Table 5 456 and 6) demonstrating the methods suitability for providing a qualifier SRM to further assure 457 selectivity as discussed later.

458 Glucagon was unstable at room temperature $(65.9 \%)$ but stable on ice $(110.1 \%)$ when 459 incubated for $4 \mathrm{hr} 50$ minutes, consistent with previous observations [31], which sufficiently 460 encompassed processing times for all batches extracted. The ability to re-inject extracts was 461 demonstrated after 42 days storage at $4^{\circ} \mathrm{C}$ (Supplementary Information Table 11). Samples 462 could be diluted 10-fold without affecting quantitation, enabling analysis in cases of low 463 sample volume (Supplementary Information Table 5).
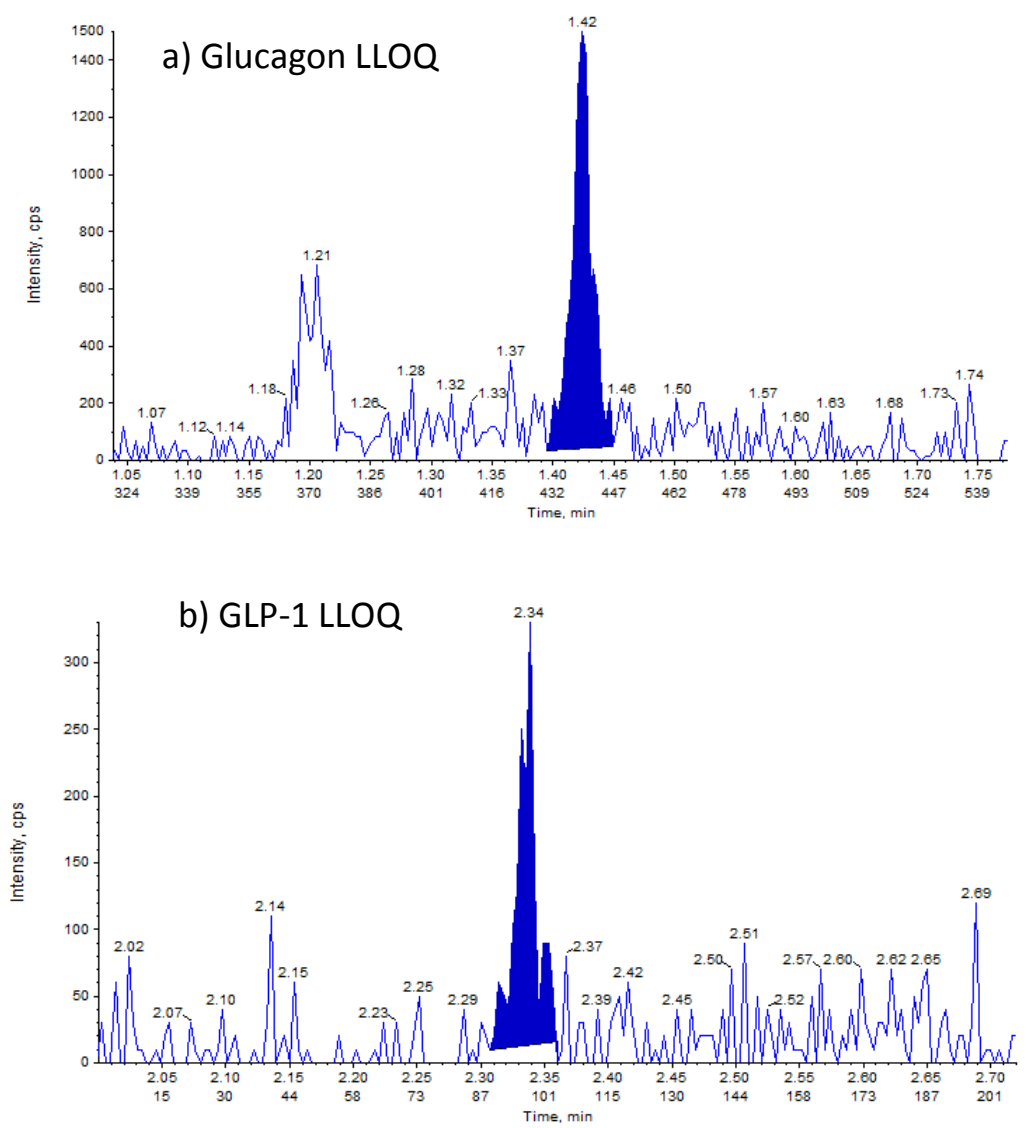
$15 \mathrm{pg} / \mathrm{mL}(697.5 / 694.0)(\mathrm{S} / \mathrm{N}=7.6)$. b) GLP-1 plasma sample at $25 \mathrm{pg} / \mathrm{mL}(660.6 / 752.0)(\mathrm{S} / \mathrm{N}=5.6)$. 
469 A SIL IS or a closely related analogue was not available for GLP-1. The glucagon internal 470 standards were therefore selected to investigate whether they offered advantages over IS 471 free quantitation for GLP-1. The GLP-1 assay was characterised for precision and accuracy 472 using two different SRM transitions for each mobile phase to ensure the most appropriate 473 was selected.

474 Precision and accuracy (\%RE and \%CV) using formic acid phases was acceptable ( $\leq 25 \%)$ 475 for all but one analyte SRM and internal standard combinations, with the 660.6/752.0 GLP-1 476 SRM with the analogue glucagon internal standard giving the best overall sensitivity and 477 performance (Supplementary Information Table 7). Signal to noise was $\geq 5$ at the LLOQ level 478 (Figure 3b). Performance was generally worse using $m$-NBA modified phases, especially at 479 the LLOQ level. This is likely due to the glucagon internal standards performing differently 480 under supercharging conditions to the GLP-1 analyte (Supplementary Information Table 7). 481 Such phases were therefore not investigated further for GLP-1.

482 GLP-1 matrix suppression was observed, whereas the analogue glucagon IS experienced 483 matrix enhancement, however the ratio between the two was similar across the matrices $484(\mathrm{CV}=16 \%)$, so there was no effect on quantitation (Supplementary Information Table 8). In 485 addition the assay gave acceptable performance across 6 individual matrices, and 6 486 replicates of lithium plasma spiked with $200 \mathrm{pg} / \mathrm{mL}$ of analyte (Supplementary Information 487 Table 7).

488 There were no interferences in the selectivity samples at the GLP-1 retention time. As 489 expected [31] GLP-1 was unstable at room temperature (9.6\%) but stable on ice for $4 \mathrm{hr} 50$ 490 minutes (94.7\%). The ability to re-inject extracts was demonstrated after 42 days storage at $4914{ }^{\circ} \mathrm{C}$ (Supplementary Information Table 11). GLP-1 recovery determined at $200 \mathrm{pg} / \mathrm{mL}$ $492(53.7 \%)$ was similar to glucagon's, and it was demonstrated that samples could be diluted 493 10-fold without affecting quantitation (Supplementary information Table 7). 
Plasma was collected from 11 healthy males and 11 healthy females and glucagon levels determined using the qualified LC-MS/MS method with standard formic acid mobile phases. Plasma glucagon concentrations were between $<15 \mathrm{pg} / \mathrm{mL}$ (BLQ) and $47.1 \mathrm{pg} / \mathrm{mL}$ (Table 1), similar to ranges reported in the literature ( $\sim 3$ to $130 \mathrm{pg} / \mathrm{mL}$ [9] or $\sim 27-87 \mathrm{pg} / \mathrm{mL}$ [20]).

Several samples contained evidence of glucagon, but the concentrations were BLQ (Figure 4). Numerical values were determined for indicative purposes by extrapolation of the calibration line, and are shown in parenthesis in Table 1. The performance of the method at the $10 \mathrm{pg} / \mathrm{mL}$ level, as determined by evaluation of surrogate matrix QCs, was reasonable (mean accuracy $87.4 \%, C V=29.7 \%$ ), however poor performance resulted at the $7.5 \mathrm{pg} / \mathrm{mL}$ level (Mean accuracy $68.6 \%, C V=40.4 \%$ ). This should be considered whilst evaluating concentrations below the established range.

507

508

509

510

511

512

513

514

515

516

517

518

519

520
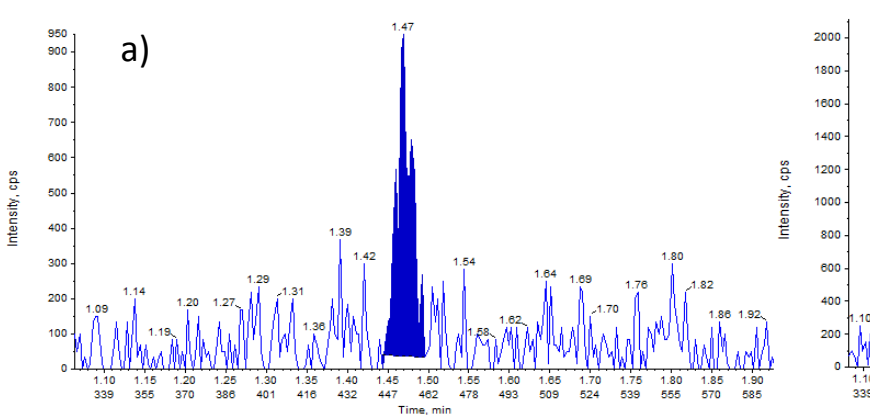

b)

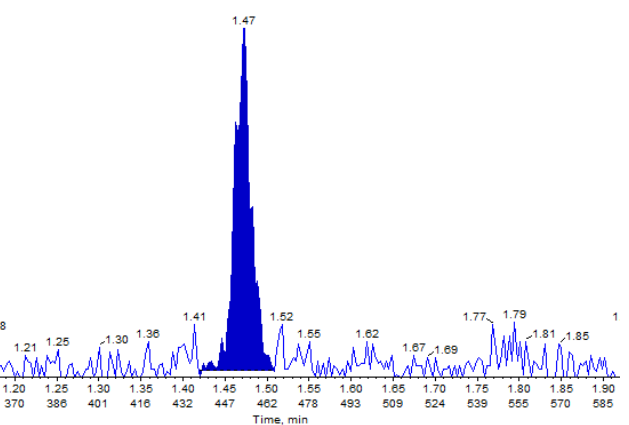

Figure 4 -Glucagon chromatograms from a a) $B L Q(14.7 \mathrm{pg} / \mathrm{mL})$ and b) within range (40.0 $\mathrm{ng} / \mathrm{mL}$ ) sample

Samples with glucagon concentrations levels above the LLOQ were reanalysed to assess incurred sample reproducibility (ISR) (Table 1). 6/9 (66.6\%) of samples gave concentrations within $20 \%$ of the original concentration, therefore passing standard criteria. A further sample gave a BLQ value upon reanalysis, although as its original concentration was close to the $15 \mathrm{pg} / \mathrm{mL}$ LLOQ, this was within the expected range. The storage time before reanalysis varied from 6 - 249 days, but no trend between the \% difference and storage time was observed.

Similarly, a pooled plasma sample ( $\mathrm{n}=6$ replicates) gave a re-assayed concentration (33.3 $\mathrm{pg} / \mathrm{mL}, \mathrm{CV}=9.4 \%)$ within $4.3 \%$ of its original concentration $(31.9 \mathrm{pg} / \mathrm{mL}, \mathrm{CV}=6.1 \%)$ following additional storage for 42 days at $-80^{\circ} \mathrm{C}$ (Supplementary information Table 9). This further demonstrated the method's good reproducibility at the endogenous glucagon level. 
Table 1 Incurred sample reproducability (ISR) assessment; comparison of original and reassayed endogenous glucagon concentrations following storage. NS- No sample remaining for repeat analysis. \% difference $=100^{*}$ (reassayed concentration- original concentration)/original concentration. Pass= \% difference within $20 \%$, Fail= \%difference outside $20 \%$.

\begin{tabular}{|c|c|c|c|c|c|c|}
\hline Sample & $\begin{array}{c}\text { Days stored } \\
\left(-80^{\circ} \mathrm{C}\right) \\
\text { before } \\
\text { original } \\
\text { analysis }\end{array}$ & $\begin{array}{c}\text { Original } \\
\text { Concentration } \\
(\mathrm{pg} / \mathrm{mL})\end{array}$ & $\begin{array}{c}\text { Days stored } \\
\text { at }-80^{\circ} \mathrm{C} \\
\text { between } \\
\text { analyses }\end{array}$ & $\begin{array}{c}\text { ISR } \\
\text { concentration } \\
(\mathrm{pg} / \mathrm{mL})\end{array}$ & $\begin{array}{c}\% \\
\text { difference }\end{array}$ & $\begin{array}{c}\text { Pass/ } \\
\text { Fail }\end{array}$ \\
\hline M1 & 95 & 19.1 & 152 & 25.3 & 27.9 & Fail \\
\hline M2 & 74 & $\mathrm{BLQ}(8.50)$ & 152 & - & - & - \\
\hline M3 & 74 & 25.7 & - & NS & - & - \\
\hline M4 & 11 & 42.8 & 152 & 39.1 & -9.0 & Pass \\
\hline M5 & 11 & 34.1 & 249 & 44.8 & 27.2 & Fail \\
\hline M6 & 11 & 27.2 & 249 & 27.8 & 2.2 & Pass \\
\hline M7 & 15 & BLQ (12.5) & - & - & - & - \\
\hline M8 & 36 & BLQ (No Peak) & - & - & - & - \\
\hline M9 & 36 & BLQ (No Peak) & - & - & - & - \\
\hline M10 & 38 & BLQ (14.1) & - & - & - & - \\
\hline M11 & 38 & 45.9 & 6 & 50.4 & 9.3 & Pass \\
\hline F1 & 95 & BLQ (14.7) & - & - & - & - \\
\hline F2 & 95 & 40.0 & 152 & 41.9 & -4.6 & Pass \\
\hline F3 & 74 & 15.1 & 152 & 15.7 & 3.9 & Pass \\
\hline F4 & 11 & 33.8 & 152 & 23.4 & -36.4 & Fail \\
\hline F5 & 11 & BLQ (No Peak) & - & - & N/A & - \\
\hline F6 & 10 & BLQ (10.3) & - & - & - & - \\
\hline F7 & 15 & BLQ (9.46) & - & - & N/A & - \\
\hline F8 & 15 & 26.9 & 99 & 24.7 & -8.5 & Pass \\
\hline F9 & 36 & BLQ (14.7) & - & - & - & - \\
\hline F10 & 38 & BLQ (14.5) & - & - & - & - \\
\hline F11 & 38 & 15.5 & 6 & BLQ (No Peak) & - & NA \\
\hline
\end{tabular}

527 For exogenous compounds the absence of significant peaks at the analyte retention time 528 can be used to demonstrate the selectivity of the method. This is not possible for 529 endogenous compounds, and therefore additional SRM transitions can be monitored as 530 qualifier transitions to ensure selectivity [16][17][18][19]. However for glucagon the only SRM 531 sensitive enough to determine endogenous concentrations using formic acid mobile phases 532 was that monitored (697.5/694.0). The method with mobile phases modified with $m$-NBA 533 enabled access to an alternative sensitive qualifier SRM transition (581.5/578.5), derived 534 from a higher charge state, as well as providing alternative LC conditions. Reanalysis of 535 extracts using this method demonstrated concentrations were not significantly different to those originally obtained providing additional evidence for the selectivity of the formic acid 
based mobile phase method (Table 2). As far as the authors knowledge the use of 538 supercharging mobile phases for such an application has not been reported.

Table 2 Determination of endogenous plasma gluagon concentrations using formic acid and $m$-NBA modified mobile phases and corresponding SRM transitions $\%$ difference $=100^{*}$

(Qualification assay concentration - quantitative assay concentration)/quantitative assay concentration. BLQ. Below the limit of quantitation (BLQ). Extrapolated concentrations given in brackets. NC- not calculatable.

\begin{tabular}{|c|c|c|c|}
\hline \multirow[t]{3}{*}{ Sample } & \multicolumn{2}{|c|}{ Glucagon Concentration (pg/mL) } & \multirow[t]{3}{*}{ \% Difference } \\
\hline & $\begin{array}{c}\text { Quantitative } \\
\text { assay }\end{array}$ & $\begin{array}{c}\text { Qualification } \\
\text { assay }\end{array}$ & \\
\hline & $\begin{array}{c}0.2 \% \text { FA Phases } \\
\text { Glucagon } \\
(697.5 / 694.0)\end{array}$ & $\begin{array}{c}0.05 \% \text { m-NBA } \\
0.01 \% \text { FA phases } \\
\text { Glucagon } \\
(581.5 / 578.5)\end{array}$ & \\
\hline F1 & BLQ (14.7) & BLQ (12.6) & -14.3 \\
\hline F2 & 40.0 & 43.3 & 8.2 \\
\hline F3 & 15.1 & 17.2 & 13.9 \\
\hline F4 & 33.8 & 32.8 & -3.0 \\
\hline F5 & No Peak & No Peak & NC \\
\hline M1 & 19.1 & 23.1 & 20.9 \\
\hline M2 & BLQ (8.50) & No Peak & NC \\
\hline M3 & 25.7 & 26.8 & 4.3 \\
\hline M4 & 42.8 & 46.2 & 7.9 \\
\hline
\end{tabular}


547 Plasma samples were available to cross-validate the LC-MS/MS method against established immunoassays. These originated from a physiological study assessing the preanalytical stability of glucagon and GLP-1 in the presence of various stabilisers and anticoagulants. These did not introduce a significant matrix effect affecting LC-MS/MS quantitation (Supplementary Information Table 10). Samples originated from fasting healthy volunteers (endogenous glucagon), volunteers dosed with glucagon, and volunteers dosed with GLP-1. Results of the stability investigations at the endogenous glucagon level have been recently reported [32]. Two immunoassays using different assay formats were used to cross-validate each analyte against the LC-MS/MS method, to help provide confidence, or otherwise, of the immunoassays results.

For endogenous glucagon quantitation, the HTRF immunoassay demonstrated limited utility as only $16 / 50$ samples were above the LLOQ $(12.0 \mathrm{pg} / \mathrm{mL})$. In contrast, the majority of the samples analysed by the Millilpex assay (38/45) and LC-MS/MS method (39/50) gave quantifiable ( $\geq$ LLOQ) concentrations. Whilst this may be expected for the LC-MS/MS method, with its lower LLOQ $(10.0 \mathrm{pg} / \mathrm{mL})$, the Milliplex had a higher LLOQ $(13.0 \mathrm{pg} / \mathrm{mL})$. This discrepancy may be due to differences in the standardisation of reference materials between the immunoassays and/or the Milliplex being more susceptible to interferences. Only data above the LLOQ is discussed below, as large errors would likely be associated with lower concentrations, although for completeness all concentrations determined are shown in Supplementary Information Figure 11a.

The endogenous glucagon concentrations measured by all three methods were within literature ranges ( 3 to $130 \mathrm{pg} / \mathrm{mL}$ [9] or $\sim 27-87 \mathrm{pg} / \mathrm{mL}$ [20]) (Figure 5 and Supplementary Figure $11 \mathrm{~b}$ ). Where both the HTRF and LC-MS/MS methods gave quantifiable ( $\geq$ LLOQ) concentrations, these were of similar magnitude with no notable inter-volunteer or anticoagulant effects (Figure 5a). Likewise the LC-MS/MS and Milliplex methods gave similar concentrations for samples from volunteers 1, 2, and 5, and from the EDTA samples from volunteer 3 (Figure 5b). However the Milliplex gave significantly higher concentrations for lithium heparin samples obtained from volunteer 3, and for all samples from volunteer 4. The Millplex assay also showed a positive bias for samples from volunteer 4 in comparison to the HTRF when quantifiable ( $\geq$ LLOQ) results were compared (Figure $5 c$ ). This suggests that the matrix from volunteer 4 affects Milliplex quantitation due to the presence of low level interferences sharing an epitope with glucagon or due to other non-specific interferences, as has been suggested previously [9] Similarly, interferences may be present in lithium heparin samples from volunteer 3 , affecting immunoassay quantitation. The poor sensitivity of the 
581

582

583

584

HTRF, and potential susceptibility of the Milliplex assay to matrix interferes, demonstrates some of the challenges in selecting appropriate immunoassays for LC-MS/MS cross validation.
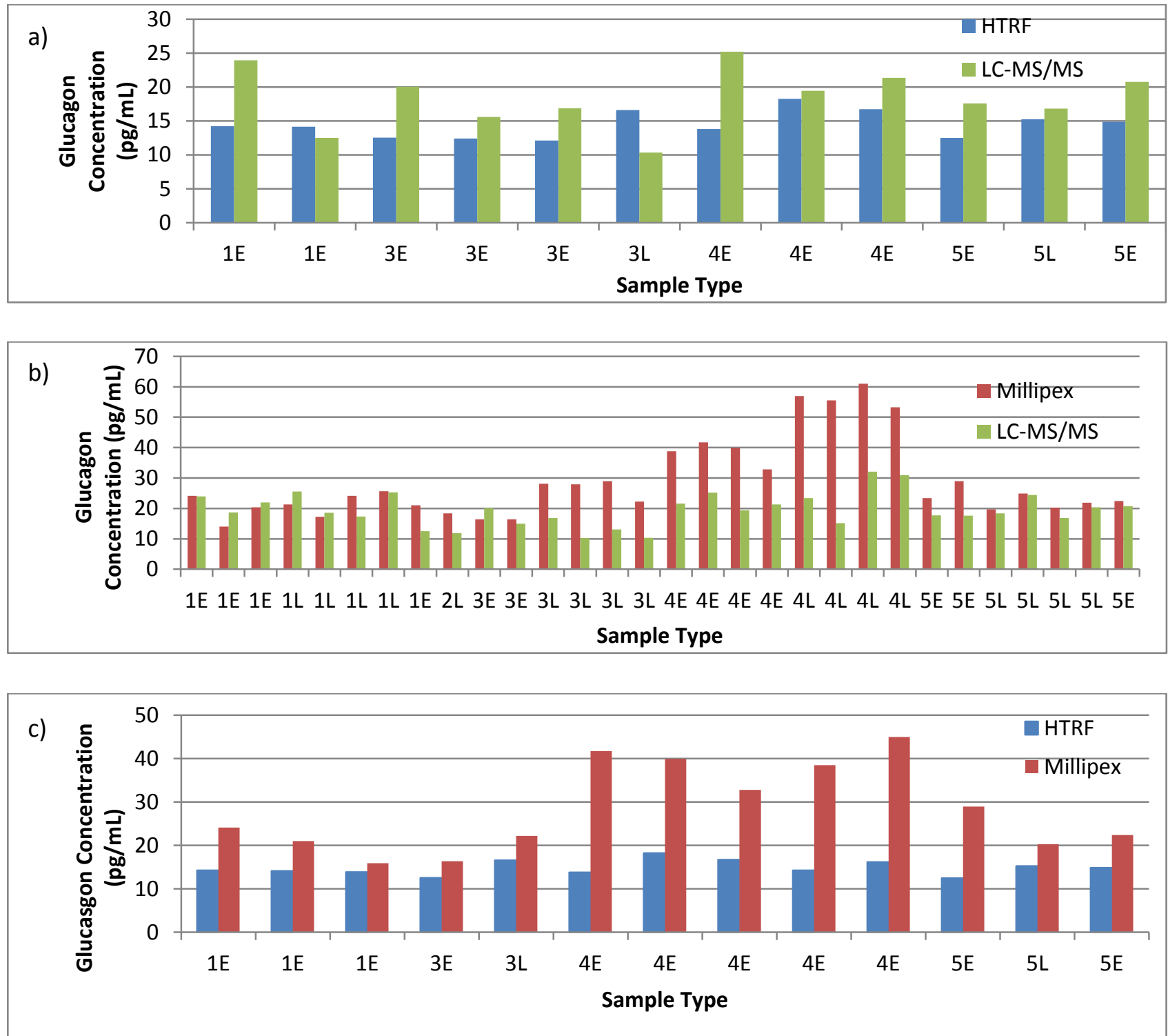

Figure 5 Comparison of endogenous glucagon concentrations determined by LC-MS/MS and two immunoassays. a) HTRF vs LC-MS/MS. b) Milliplex vs. LCMS/MS, c) HTRF vs. Milliplex. Samples were analysed from 5 volunteers (1-5), which contained either EDTA (E) or Lithium Heparin (L) anticoagulant in addition to various stabilisers (not shown). Different stabiliser combinations were used in samples of the same Sample Type, e.g. the four 4E samples in Figure $5 \mathrm{~b}$ are all unique. Only samples with quantifiable ( $\geq L L O Q$ ) results for both assays being compared are displayed. Full results are shown in Supplementary Information Figure 
Concentrations of the samples from the volunteer dosed with glucagon strongly correlated between the two immunoassays, and similar absolute concentrations were determined 600 (Figure 6a). This may be due to the absence of matrix interferences affecting the Milliplex 601 assay from this volunteer, or interferences becoming negligible at higher glucagon concentrations. The LC-MS/MS method showed some similarity in concentrations trends between samples, although results were less comparable, and absolute concentrations were generally higher. This may reflect differences in the natures of the techniques; immunoassays detect specific epitopes whereas the LC-MS/MS method targets the whole molecule. It has been recently suggested that epitope masking caused by glucagon aggregation may artificially lower immunoassay assay concentrations in plasma samples fortified with high levels of glucagon [26]. A similar process may occur in samples from dosed patients also containing high levels of glucagon. In addition to generally higher LCMS/MS concentrations, this could also explain differences in concentration trends if aggregation occurred to various degrees in different samples.
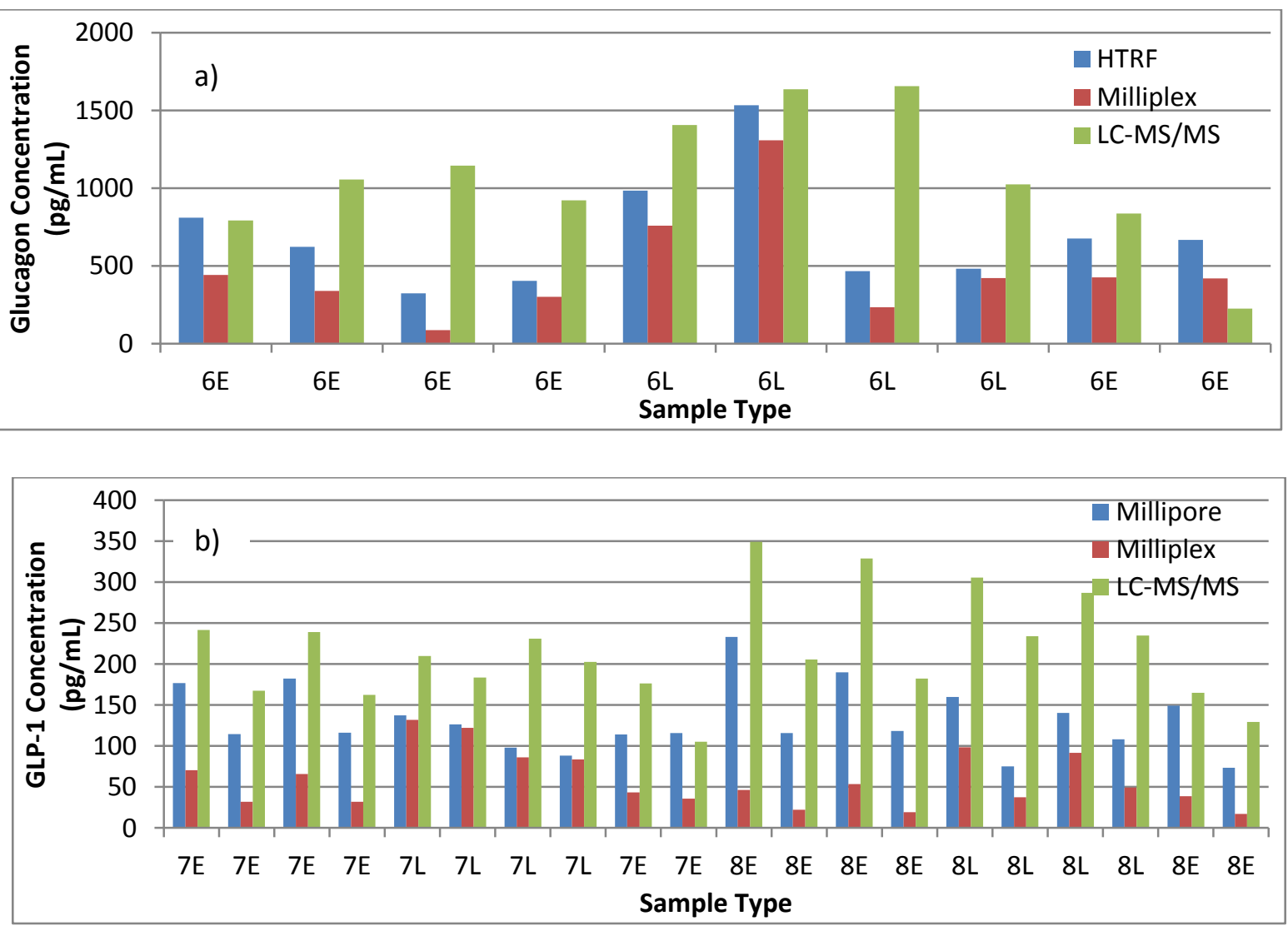
GLP-1. Samples were analysed from 1 volunteer (labelled 6) dosed with glucagon and 2 volunteers (labelled 7 \& 8) dosed with GLP-1. Samples contained either EDTA

(E) or Lithium Heparin (L) anticoagulant in addition to various stabilisers (not shown). Different stabiliser combinations were used in samples of the same Sample Type, e.g the six $6 \mathrm{E}$ samples are all unique. 
621 Samples from the two volunteers dosed with GLP-1 showed similar concentrations trends 622 between the samples across the three methods (Figure 6b). Absolute concentrations 623 showed a systematic bias of LC-MS/MS > Millpore> Milliplex. This could be due to 624 differences in the standardisation of reference materials. Although it was also noted that 625 lithium heparin samples from subject 7 showed similar immunoassay concentrations, 626 suggesting a susceptibility of the assays to inter-volunteer and anticoagulant related matrix 627 effects. The limited agreement of the immunoassays restricted their value to further cross628 validate the LC-MS/MS method.

629 Overall, the cross-validation provided only limited confirmation for the performance of the 630 LC-MS/MS method. However this was not unexpected as the poor performance of some 631 immunoassays provided the main rationale for development of the LC-MS/MS method. 632 Further investigations are needed with larger data sets, various sample types, and additional 633 assays, to better characterise the performance of the developed LC-MS/MS method. 
We describe a multiplexed LC-MS/MS method for the quantitation of endogenous glucagon, dosed glucagon, and/or dosed GLP-1 in human plasma without immunoenrichment. The sensitivity achieved is the highest reported for both peptides for such an extraction. The methodology has been improved over the initial method through a significant shortening of extraction time, inclusion of a stable isotope labelled internal standard and a reduction in LCMS cycle time. The one day extraction compares well against many immunoassays, which often incur overnight incubation steps, as required by the Millipore and Millipex assays used in this study. Samples extracted for LC-MS/MS analysis can be run overnight to produce data is a similar overall time frame to immunoassays. Good precision and accuracy was obtained over the calibration range, and recovery, matrix effects, and bench top stability were acceptable. Endogenous glucagon specificity was assured by a novel approach using mobile phases modified with the $m$-NBA supercharging additive to access an alternative sensitive qualifier SRM transition.

Endogenous glucagon concentrations were within the expected range, and determination was highly reproducible after extended sample storage (up to 249 days at $-80^{\circ} \mathrm{C}$ ). A crossvalidation was performed using physiological samples with the qualified LC-MS/MS method and two immunoassays for each analyte. Some similarities between the LC-MS/MS method and immunoassays were observed for endogenous glucagon, but dosed glucagon and dosed GLP-1 samples showed a trend towards higher concentrations when measured by LC-MS/MS. It was however noted that immunoassays also showed some differences between each other, limiting their value for cross validation. Such differences were not wholly unexpected, as performance concerns associated with glucagon and GLP-1 immunoassays provided the main rationale for development of the LC-MS/MS method. We therefore consider the LC-MS/MS method to be at viable alternative to immunoassay based approaches.

However we appreciate that cost may be a limitation to the adoption of LC-MS/MS to routine clinical diagnostics in some laboratories. Whilst consumable costs are lower, with the largest being the SPE plate at a couple of hundred pounds (compared to several hundred pounds for immunoassays kits), LC-MS/MS instrumentation is considerably more expensive. Sensitive LC-MS/MS systems cost in the region of two hundred thousand pounds, whereas plate readers can be obtained for around 10 times less. Although as a platform technology LC-MS/MS enables rapid amortisation of the capital cost over multiple analytes. The uptake of LC-MS/MS within clinical laboratories is increasing, and therefore there is the potential to 
669 initiate specialist centres with access to LC-MS/MS instrumentation, who could set up and 670 run a glucagon LC-MS/MS service.

\section{$671 \quad$ Future Perspectives}

672 As instrumentation improves it will be possible to accurately quantity lower levels of 673 endogenous glucagon as well as endogenous GLP-1 without the use of immunoenrichment.

674 These will also allow sensitive qualifier SRM transitions to be obtained using standard formic 675 acid mobile phases to ensure endogenous compound specificity, until then transitions may 676 be accessed using mobile phase supercharging additives as described. The use of high 677 throughput 2D extractions to minimise matrix suppression, may also become a more 678 widespread strategy to improve sensitivity. Further development could also include the 679 expansion of the assay to other proglucagon derived peptides, or other peptides of interest. 680 Reproduction of LC-MS/MS methods at different laboratories will provide further confidence 681 of the performance and robustness of these.

682 The differences in glucagon and GLP-1 concentrations obtained using the various analytical 683 methods is concerning, as they could lead to incorrect clinical decisions. Assays should be 684 better characterised with regards to inter-subject and anticoagulant related matrix 685 interferences, as well as for cross-reactivity with metabolites and degradation fragments. 686 Characterisation may include the use of biological matrix reference materials containing 687 independently certified amounts of analyte [33], but these are not currently available for 688 glucagon or GLP-1. The ability of glucagon to aggregate in plasma, and its effect on 689 quantitation, requires further study, and the standardisation of reference materials between 690 assays should be improved. The benefit of using LC-MS/MS based methodologies is that 691 specificity is enhanced over immunoassays, as they can target the full length molecule 692 rather than specific epitopes, and therefore such methods may become increasingly utilised 693 in the future. 
695

696

697

698

699

700

701

702

703

704

705

706

707

708

709

710

711

712

713

714

715

\section{Executive Summary}

\section{Background}

- Glucagon and GLP-1 are traditionally quantified in human plasma by immunoassay. However precision and accuracy can be poor, as can be the correlation between assays.

- LC-MS/MS based approaches can be used as alternatives to help overcome such challenges; however their application has been limited by lower sensitivity.

- This study refined and expanded our previous glucagon-only method, improving sensitivity, robustness, and throughput, and further demonstrated specificity of endogenous glucagon quantitation. GLP-1 was also introduced as a secondary analyte.

\section{Experimental}

- Method refinements included modification of the 2D (protein precipitation then SPE) extraction protocol and the introduction of an automated liquid handling system to improve sensitivity, robustness, and throughout.

- The multiplexed method was qualified based on key experiments described in the latest FDA and EMA bioanalytical guidelines.

- A sensitive qualifier SRM was accessed via a novel application of a supercharging mobile phase additive to ensure specificity of endogenous glucagon quantitation.

- Physiological study samples were quantified using the qualified LC-MS/MS assay, and a cross-validation was performed against established immunoassays. 
- Method refinements halved the extraction time to 1 working day (96 samples) and UHPLC run time to 3.6 minutes/samples, significantly improving throughput. Sensitivity was improved by reduced matrix suppression in the refined method.

- The method was successfully multiplexed with GLP-1, and met the qualification criteria for both analytes.

- Endogenous glucagon concentrations were determined within the expected range, and confirmed by comparison to a sensitive qualifier SRM. Concentrations showed good reproducibility after extended sample storage.

- Glucagon and GLP-1 concentrations were determined from physiological study samples containing endogenous glucagon, dosed glucagon and dosed GLP-1.

- Cross-validation against immunoassays using the physiological study samples showed some similarities between the methods. However differences were not wholly unexpected, as performance concerns associated with glucagon and GLP-1 immunoassays provided the main rationale for development of the LC-MS/MS method.

\section{Conclusion}

- The sensitivity achieved (Glucagon- $15 \mathrm{pg} / \mathrm{mL}$ LLOQ, GLP-1- $25 \mathrm{pg} / \mathrm{mL}$ LLOQ) is the highest reported for both peptides for an extraction avoiding immunoenrichment

- The method provides a viable alternative to immunoassay based approaches for the quantitation of endogenous glucagon, dosed glucagon, and/or dosed GLP-1 from human plasma samples.

\section{Financial \& competing interests disclosure}

The authors have no relevant affiliations or financial involvement with any organization or entity with a financial interest in or financial conflict with the subject matter or materials discussed in the manuscript. This includes employment, consultancies, honoraria, stock ownership or options, expert testimony, grants or patents received or pending, or royalties.

No writing assistance was utilized in the production of this manuscript. 


\section{$748 \quad$ Ethical conduct of research}

749 The authors state that they have obtained appropriate institutional review board approval or

750 have followed the principles outlined in the Declaration of Helsinki for all human or animal 751 experimental investigations. In addition, for investigations involving human subjects,

752 informed consent has been obtained from the participants involved.

\section{$753 \quad$ References of interest}

754 [10]—Comprehensive overview of immunological approaches for the quantitation of the 755 various GLP-1 isoforms, including practical considerations.

756 [28] Description of the mechanism via which supercharging molecules lead to increased 757 analyte charging.

758 [30]- Evaluation of various strategies for endogenous quantitation.

\section{References of considerable interest}

760 [9] - All seven glucagon immunoassays evaluated demonstrated poor performance in terms

761 of specificity, spiked recovery, susceptibility to matrix effects/non-specific matrix

762 interferences and/or and sensitivity.

763 [11]- Considerable variability in specificity and sensitivity was observed between the ten 764 GLP-1 immunoassays kits evaluated.

765 [20] -An Immunoaffinity extraction with microflow LC was used to develop the most sensitive 766 glucagon (2.7 pg/mL LLOQ ) and GLP-1 (2.6 pg/mL LLOQ) LC-MS/MS method in the 767 literature. 


\section{References}

[1] Sandoval D a, D'Alessio D a. Physiology of Proglucagon Peptides: Role of Glucagon and GLP-1 in Health and Disease. Physiol. Rev. 95(2), 513-548 (2015).

[2] Kolb A, Rieder S, Born D, et al. Glucagon/insulin ratio as a potential biomarker for pancreatic cancer in patients with new-onset diabetes mellitus. Cancer Biol. Ther. 8(16), 1527-1533 (2009).

[3] Furuse M, Matsumoto M, Okumura JI, Sugahara K, Hasegawa S. Intracerebroventricular injection of mammalian and chicken glucagon-like peptide-1 inhibits food intake of the neonatal chick. Brain Res. 755(1), 167-169 (1997).

[4] Holst JJ. The Physiology of Glucagon-like Peptide 1. Physiol. Rev. (225), 1409-1439 (2007).

[5] De Graaf C, Blom WAM, Smeets PAM, Stafleu A, Hendriks HFJ. Biomarkers of satiation and satiety. Am. J. Clin. Nutr. 79(6), 946-961 (2004).

[6] Parker $\mathrm{J}$ a, McCullough $\mathrm{K}$ a, Field BCT, et al. Glucagon and GLP-1 inhibit food intake and increase c-fos expression in similar appetite regulating centres in the brainstem and amygdala. Int. J. Obes. (Lond). 37(10), 1391-8 (2013).

[7] Tan TM, Field BCT, McCullough $\mathrm{K}$ a, et al. Coadministration of glucagon-like peptide1 during glucagon infusion in humans results in increased energy expenditure and amelioration of hyperglycemia. Diabetes. 62(4), 1131-8 (2013).

[8] Alford FP, Bloom SR, Nabarro JD. Glucagon levels in normal and diabetic subjects: Use of a specific immunoabsorbent for glucagon radioimmunoassay. Diabetologia. 13(1), 1-6 (1977).

[9] Bak MJ, Albrechtsen NW, Pedersen J, et al. Specificity and sensitivity of commercially available assays for glucagon and oxyntomodulin measurement in humans. Eur J Endocrino. 170(4), 529-38 (2014).

[10] Kuhre RE, Wewer Albrechtsen NJ, Hartmann B, Deacon CF, Holst JJ. Measurement of the incretin hormones: Glucagon-like peptide-1 and glucose-dependent insulinotropic peptide. J. Diabetes Complications. 29(3), 445-450 (2015).

[11] Bak MJ, Albrechtsen NJW, Pedersen J, et al. Specificity and sensitivity of commercially available assays for glucagon-like peptide-1 (GLP-1): implications for GLP-1 measurements in clinical studies. Diabetes, Obes. Metab. 16, 1155-1164 (2012).

[12] Heijboer AC, Frans A, Lomecky M, Blankenstein MA. Analysis of glucagon-like peptide 1; what to measure? Clin. Chim. Acta. 412(13-14), 1191-4 (2011).

[13] Veniamin N Lapko, Patrick S Miller, G Paul Brown, Rafiqul Islam, Sarah K Peters, Richard L Sukovaty PFR\& CJK. Sensitive glucagon quantification by immunochemical and LC - MS / MS methods. Bioanalysis. 5(23), 2957-2972 (2013).

[14] Ørskov C, Rabenhøj L, Wettergren A, Kofod H, Holst JJ. Tissue and Plasma Concentrations of Amidated and Glycine-Extended Glucagon-Like Peptide I in Humans. Diabetes. 43(4), 535-539 (1994).

[15] Howard JW, Kay RG, Tan T, Minnion J, Creaser CS. Identification of plasma protease derived metabolites of glucagon and their formation under typical laboratory 
[16] Honour JW. Development and validation of a quantitative assay based on tandem mass spectrometry. Ann Clin Biochem. 48(2), 97-111 (2011).

[17] Abbatiello SE, Mani DR, Keshishian H, Carr SA. Automated detection of inaccurate and imprecise transitions in peptide quantification by multiple reaction monitoring

[22] Chappell D, Lee A, Castro-Perez J, et al. An ultrasensitive method for the quantitation of active and inactive GLP-1 in human plasma via immunoaffinity LCMS/MS. Bioanalysis. 6(1), 33-42 (2014).

[23] Amao M, Kitahara Y, Tokunaga A, Shimbo K, Eto Y, Yamada N. Simultaneous quantification of intracellular and secreted active and inactive glucagon-like peptide-1 from cultured cells. Anal. Biochem. 472, 45-51 (2015).

[24] Guidance for industry: Bioanalytical method validation. U.S. Department of Health and Human Services, Food and Drug Administration, Center for Drug Evaluation and Research (CDER), Center for Veterinary Medicine (CVM), May 2001, (2001).

[25] Guideline on bioanalytical method validation, EMA(2012).

[26] Wewer Albrechtsen NJ, Bak MJ, Hartmann B, et al. Stability of glucagon-like peptide 1 and glucagon in human plasma. Endocr. Connect. 4(1), 50-57 (2015).

[27] Kay RG, Howard J, Stensson S. A current perspective of supercharging reagents and peptide bioanalysis. Bioanalysis. 8(3), 157-161 (2016).

[28] lavarone AT, Williams ER. Mechanism of charging and supercharging molecules in electrospray ionization. J. Am. Chem. Soc. 125(8), 2319-27 (2003).

[29] Houghton R, Horro Pita C, Ward I, Macarthur R. Generic approach to validation of small-molecule LC-MS/MS biomarker assays. Bioanalysis. 1(8), 1365-74 (2009).

[30] Jones BR, Schultz G a, Eckstein J a, Ackermann BL. Surrogate matrix and surrogate analyte approaches for definitive quantitation of endogenous biomolecules. Bioanalysis. 4(19), 2343-56 (2012).

[31] Deacon C, Johnsen A, Holst J. Degradation of glucagon-like peptide-1 by human plasma in vitro yields an $\mathrm{N}$-terminally truncated peptide that is a major endogenous metabolite in vivo. J Clin Endocrinol Metab. 80(3), 925-7 (1995). 
853

854

855

856

857

858

859
[32] Cegla J, Jones BJ, Howard J, et al. The preanalytical stability of glucagon as measured by liquid chromatography tandem mass spectrometry and two commercially available immunoassays. Ann. Clin. Biochem.(2016).

[33] French D. Development and validation of a serum total testosterone liquid chromatography-tandem mass spectrometry (LC-MS/MS) assay calibrated to NIST SRM 971. Clin. Chim. Acta. 415, 109-117 (2013). 
5 Table S1 Parameters re-optimised during assay development for glucagon quantitation

\begin{tabular}{|c|c|c|c|c|c|}
\hline \multicolumn{2}{|c|}{ Parameter Re-optimised } & \multirow{2}{*}{$\begin{array}{l}\text { Aim } \\
\text { Increase sensitivity by increasing recovery and/or } \\
\text { reducing matrix effects }\end{array}$} & \multirow{2}{*}{$\begin{array}{l}\text { Result } \\
\text { Original solvent }\left(75 / 25 / 0.1, \mathrm{ACN} / \mathrm{H}_{2} \mathrm{O} / \mathrm{NH}_{3}\right) \text { gave } \\
\text { optimal performance }\end{array}$} & \multirow{2}{*}{$\begin{array}{l}\text { Method } \\
\text { altered? } \\
\text { No }\end{array}$} & \multirow{2}{*}{$\begin{array}{l}\text { Sup } \\
\text { Info } \\
\text { Ref }\end{array}$} \\
\hline \multirow{3}{*}{$\begin{array}{l}\text { Protein } \\
\text { Precipitation } \\
\text { Solvent }\end{array}$} & Composition & & & & \\
\hline & Volume & $\begin{array}{l}\text { Reduce extraction time by reducing solvent volume to } \\
\text { enable use of } 96 \text { well plates and associated } \\
\text { automated liquid handling, as well as reducing } \\
\text { evaporation time. }\end{array}$ & $\begin{array}{l}\text { Volume reduced from } 3.2 \mathrm{~mL} \text { to } 1.1 \mathrm{~mL} \text {. } \\
\text { Automated liquid handling utilised. Extraction time } \\
\text { halved to } 1 \text { day. }\end{array}$ & Yes & 1.2 \\
\hline & $\begin{array}{l}\text { Evaporation } \\
\text { temperature }\end{array}$ & Reduce evaporation time & $\begin{array}{l}\text { Higher temperatures }\left(60^{\circ} \mathrm{C}\right) \text { led to increased } \\
\text { matrix interference. }\end{array}$ & No & 1.3 \\
\hline \multicolumn{2}{|c|}{ Extract Reconstitution Volume } & $\begin{array}{l}\text { Increase sensitivity by reducing reconstitution volume } \\
\text { to increase extract concentration }\end{array}$ & $\begin{array}{l}\text { Reduced volume increases background noise, } \\
\text { lowering signal-to-noise. }\end{array}$ & No & 2 \\
\hline \multirow{3}{*}{ LC } & Flow Rate & $\begin{array}{l}\text { Increase sensitivity, as a result of higher ionisation } \\
\text { efficiencies at lower flow rates. Monitor multiple charge } \\
\text { states to ensure optimal is selected. }\end{array}$ & $\begin{array}{l}\text { No overall boost in sensitivity was observed due } \\
\text { to increased peak broadening at lower flow rates. } \\
\text { Original charge state was optimal. }\end{array}$ & No & 3.1 \\
\hline & $\begin{array}{l}\text { Formic acid } \\
\text { composition }\end{array}$ & $\begin{array}{l}\text { Increase sensitivity, by increasing positive electrospray } \\
\text { ionisation at lower } \mathrm{pH}\end{array}$ & Sensitivity reduces at lower $\mathrm{pH}$. & No & 3.2 \\
\hline & $\begin{array}{l}\text { Column clean } \\
\text { time }\end{array}$ & $\begin{array}{l}\text { Reduce run time by reducing column clean, enabled } \\
\text { by lower matrix build up by automated liquid handling. }\end{array}$ & $\begin{array}{l}\text { Column clean time reduced by } 3 \text { minutes, leading } \\
\text { to a gradient time of } 3.6 \text { min per sample, saving } 5 \\
\text { hours of analysis time per } 96 \text { sample batch. }\end{array}$ & Yes & 3.3 \\
\hline \multicolumn{2}{|c|}{ Internal Standard } & $\begin{array}{l}\text { Increase precision and accuracy, and robustness } \\
\text { using SIL rather than an analogue glucagon IS }\end{array}$ & $\begin{array}{l}\text { SIL IS gave better performance under conditions } \\
\text { of high matrix effect. }\end{array}$ & Yes & $2 \& 4$ \\
\hline
\end{tabular}




\section{Extraction Optimisation}

\section{$7 \quad 1.1 \quad$ Protein Precipitation Solvent Composition}

8 Previously we investigated ACN based protein precipitation solvents diluted to various 9 proportions $(0,25,50 \%)$ with water [1]. We found that $75 / 25 \mathrm{ACN} / \mathrm{H}_{2} \mathrm{O}$ gave the best response, and that the addition of $0.1 \%$ formic acid significantly reduced it, whilst the addition $0.1 \%$ ammonia gave a small increase. Therefore 75/25/0.1 $\mathrm{ACN} / \mathrm{H}_{2} \mathrm{O} / \mathrm{NH}_{3}$ was selected. To attempt to increase performance further in this sutdy we investigated additional proportions of $\mathrm{ACN}$ in water $(20,15,10,5 \%)$ and investigated the use of $\mathrm{MeOH}$ based solvents, however the original solvent remained optimal (Figure S1). We have found in our laboratory that using $25 \%$ water in acetonitrile is often the optimal protein precipitation solvent for peptide analysis.

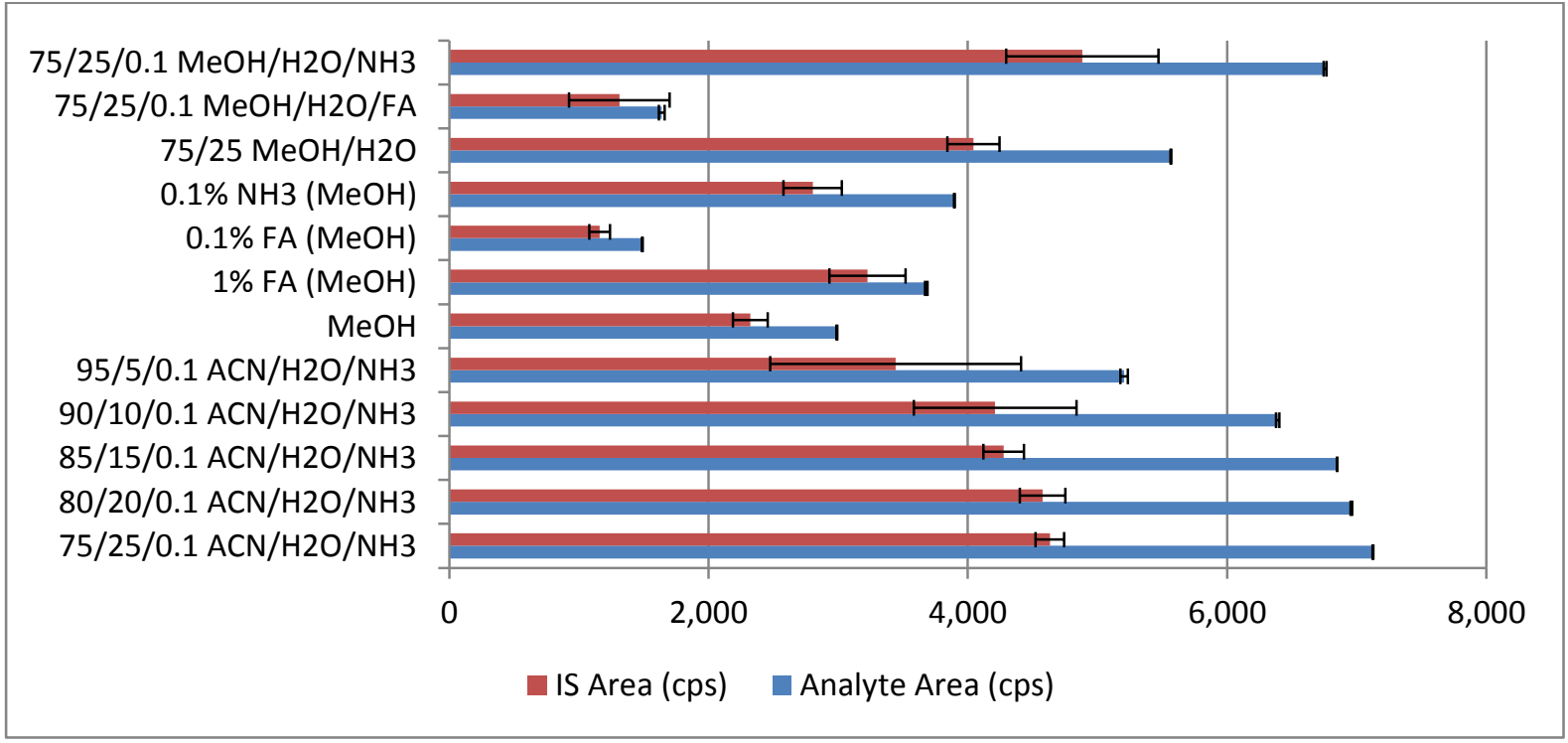
extracting plasma samples $(100 \mu \mathrm{L})$ spiked with $1 \mathrm{ng} / \mathrm{mL}$ glucagon with various protein precipitation solvents 

to glucagon's retention time (1.7 min) were eliminated (Figure S2 a vs b).

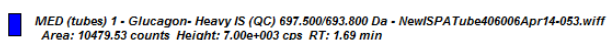

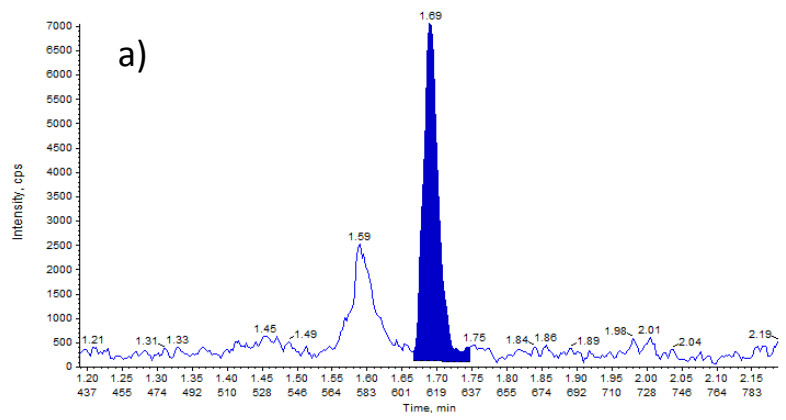

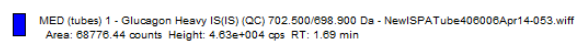

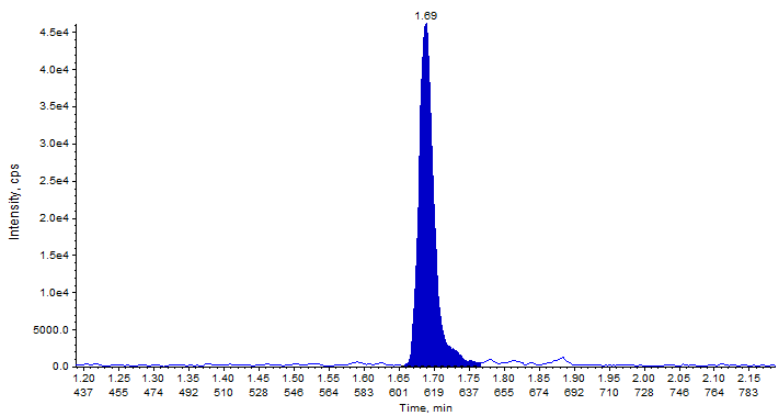

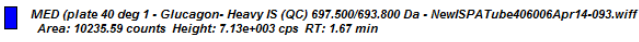

\section{1}
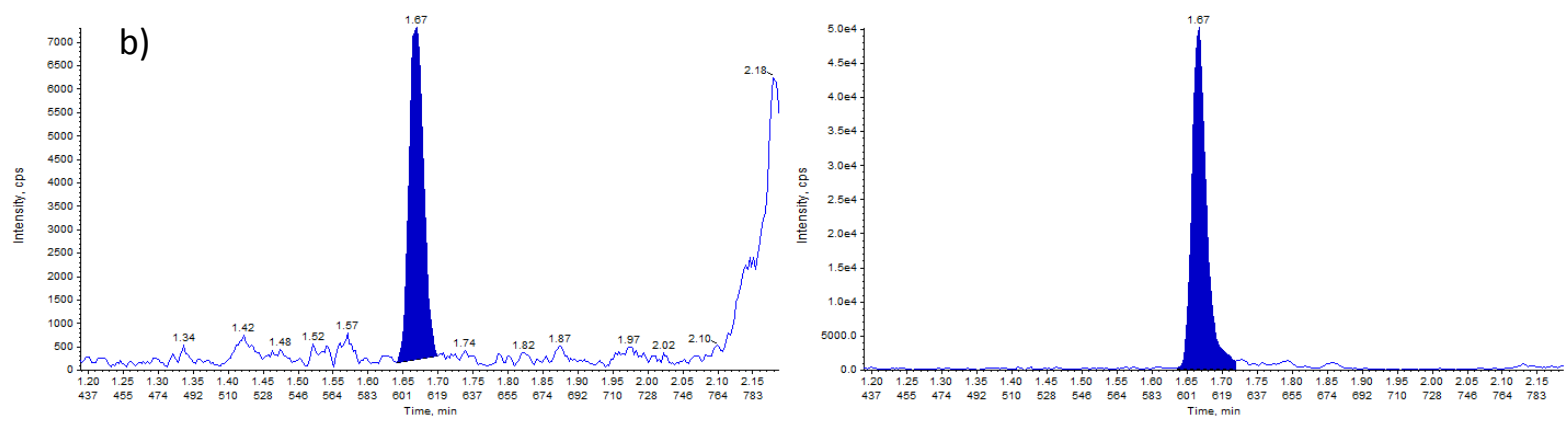

27

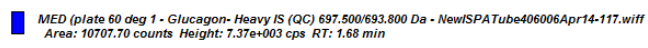

\footnotetext{
1
}
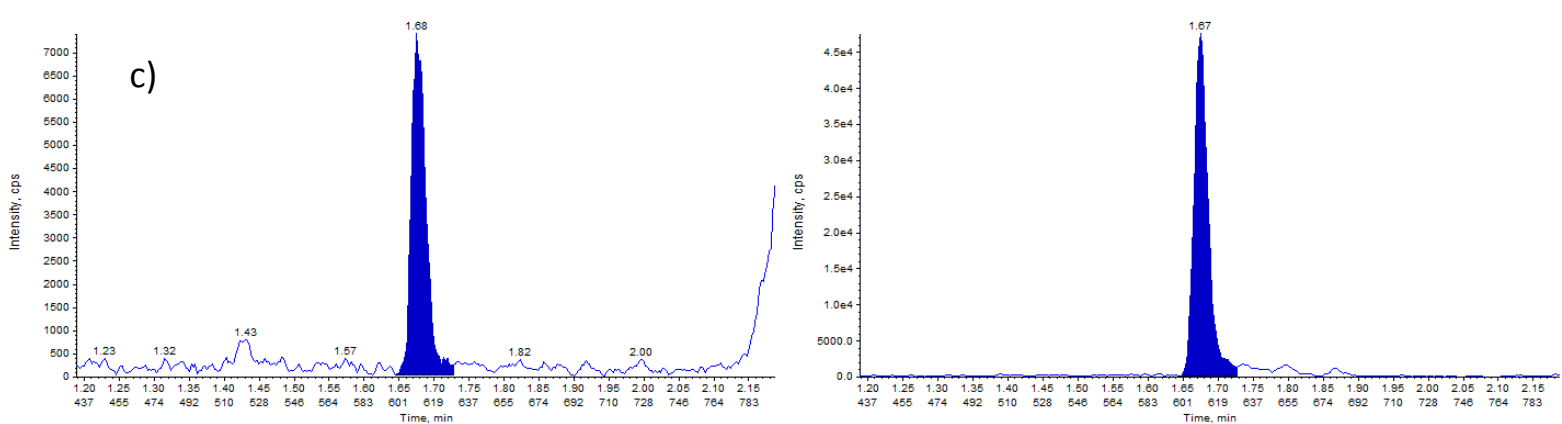

Figure S2 Effect of the extraction method on interference peaks. The matrix interference (1.6 $\mathrm{min}$ ) close to the analyte retention time observed using the a) original extraction procedure is removed using the b) modified extraction procedure. c) Increasing the solvent evaporation temperature to $60^{\circ} \mathrm{C}$ resulted in a slight decrease in internal standard response and an increase in tailing. 


\subsection{Protein Precipitation Solvent Evaporation Temperature}

The blow down temperature was increased from $40^{\circ} \mathrm{C}$ to $60^{\circ} \mathrm{C}$ to attempt to reduce evaporation time. No significant difference in analyte signal (Figure S2 b vs c) or QC accuracies were observed, however the IS showed slightly lower signal and greater tailing. As the difference in evaporation time was small (75 $\mathrm{min}$ vs $95 \mathrm{~min}$ ), it was decided to maintain the temperature at $40^{\circ} \mathrm{C}$.

\section{Extract Reconstitution Volume}

Lower extract reconstitution volumes were investigated to attempt to boost sensitivity. However whilst signal did increase the background noise increased to a greater extent, leading to a reduction in signal-to-noise with a constant $40 \mu \mathrm{L}$ injection volume $(18.4,15.5$, 12.9 , at $200,150,100 \mu \mathrm{L}$ reconstitution volumes respectively for a $100 \mathrm{pg} / \mathrm{mL}$ sample).

The Analye/internal standard peak-area-ratio (PAR) was also affected at low reconstitution volumes $(100 \mu \mathrm{L})$ using the analogue internal standard for 100 and $750 \mathrm{pg} / \mathrm{mL}$ samples, affecting quantitative accuracy and demonstrating the limitation of the analogue internal standard under conditions of high matrix effects (Table 2).

Table 2 Effect of reconstitution volume on extracted plasma QC performance, quantified using the analogue and SIL glucagon internal standard.

\begin{tabular}{|c|c|c|c|c|c|c|c|c|}
\hline \multirow{4}{*}{$\begin{array}{l}\text { Reconstitution } \\
\text { volume }(\mu \mathrm{L})\end{array}$} & \multicolumn{8}{|c|}{ Glucagon Internal Standard } \\
\hline & \multicolumn{4}{|c|}{ Analogue } & \multicolumn{4}{|c|}{ SIL } \\
\hline & \multicolumn{2}{|c|}{$100 \mathrm{pg} / \mathrm{mL}$} & \multicolumn{2}{|c|}{$750 \mathrm{pg} / \mathrm{mL}$} & \multicolumn{2}{|c|}{$100 \mathrm{pg} / \mathrm{mL}$} & \multicolumn{2}{|c|}{$750 \mathrm{pg} / \mathrm{mL}$} \\
\hline & $\begin{array}{c}\% 200 \mu \mathrm{L} \\
\text { PAR }\end{array}$ & $\% \mathrm{CV}$ & $\begin{array}{c}\% 200 \mu \mathrm{L} \\
\text { PAR }\end{array}$ & $\% \mathrm{CV}$ & $\begin{array}{c}\% 200 \mu \mathrm{L} \\
\text { PAR }\end{array}$ & $\% \mathrm{CV}$ & $\begin{array}{c}\% 200 \mu \mathrm{L} \\
\text { PAR }\end{array}$ & $\% \mathrm{CV}$ \\
\hline 200 & 100.0 & 2.0 & 100.0 & 5.7 & 100.0 & 1.6 & 100.0 & 4.1 \\
\hline 150 & 97.7 & 1.7 & 99.8 & 0.2 & 103.7 & 1.6 & 102.8 & 1.1 \\
\hline 100 & 78.1 & 3.6 & 89.8 & 6.9 & 98.5 & 1.3 & 104.3 & 2.6 \\
\hline
\end{tabular}

\section{LC Optimisation}

\subsection{Flow Rate}

Lower LC-flow rates $(0.25 \mathrm{~mL} / \mathrm{min}$ and $0.40 \mathrm{~mL} / \mathrm{min})$ were investigated to see whether they increased sensitivity over the original $(0.80 \mathrm{~mL} / \mathrm{min})$ flow rate, due to improved ionisation efficiency. To reduce the effect of peak broadening flow rates were lowered only during analyte elution.

Lower flow rates were noted to increase the relative formation of the highly charged $4+$ ion ( $m / z=697)$, as expected due to prolonged interaction with the electrospray probe allowing greater charge accumulation. Peak areas increased with lower flow rates (Figure S3b), however peak broadening was still significant, and therefore there was no overall significant increase in peak height (sensitivity) (Figure S3b). The flow rate was maintained at 0.8 $\mathrm{mL} / \mathrm{min}$. 

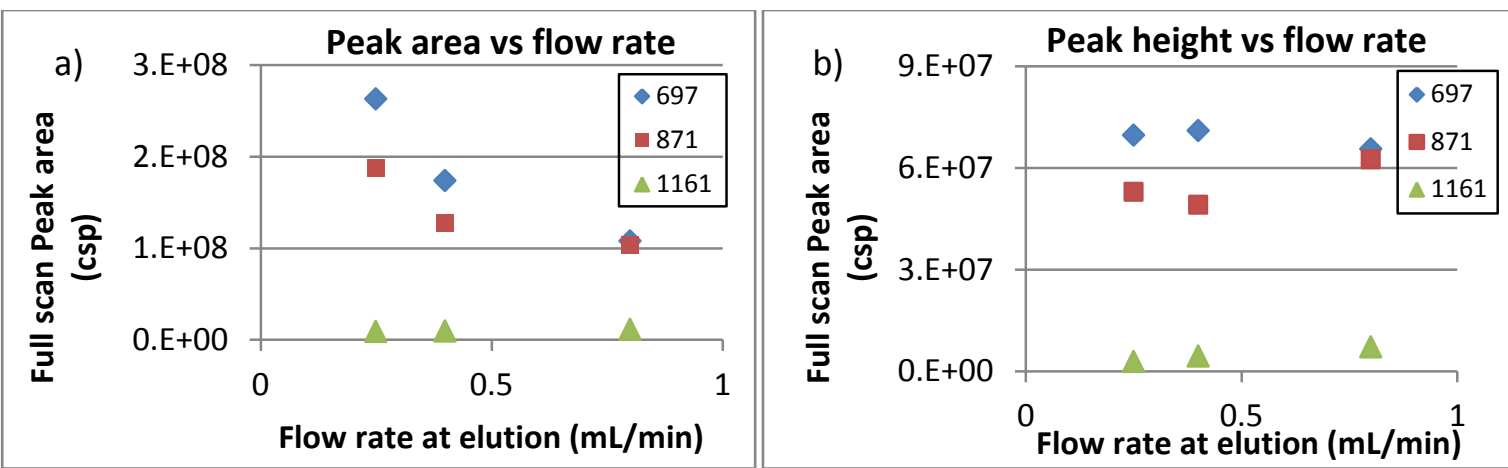

Figure S3 Effect of the flow rate on (a) peak area and (b) heights for various glucagon charge states using $1 \mathrm{ng} / \mathrm{mL}$ solution. Flow rate is $0.8 \mathrm{~mL} / \mathrm{min}$ except at elution. Mobile phases $A=$ $0.2 \% \mathrm{FA}(\mathrm{ACN}) \mathrm{B}=0.2 \% \mathrm{FA}(\mathrm{aq})$.

\subsection{Formic Acid Composition}

The percentage of formic acid in the mobile phases was investigated using $1 \mathrm{ng} / \mathrm{mL}$ glucagon solutions (Figure S4). Reducing formic acid from $1 \%$ to $0.2 \%$ increased the intensity of all charge states, including the most highly charged $5+(\mathrm{m} / \mathrm{z}=697)$ charge state, without significantly affecting the charge state distribution. Higher percentages of formic acid may increase ionisation of matrix components, limiting ion current available for analyte ions. However the proportion of the $5+(m / z=697)$ charge state reduced in abundance when formic acid was lowered further to $0.1 \%$, as the lower $4+(\mathrm{m} / \mathrm{z}=871)$ charge state was favoured. Peaks height and peak area were similarly affected, as there was not effect on peak broadening. $0.2 \%$ FA remains optimal for the $5+(m / z=697)$ charge state.
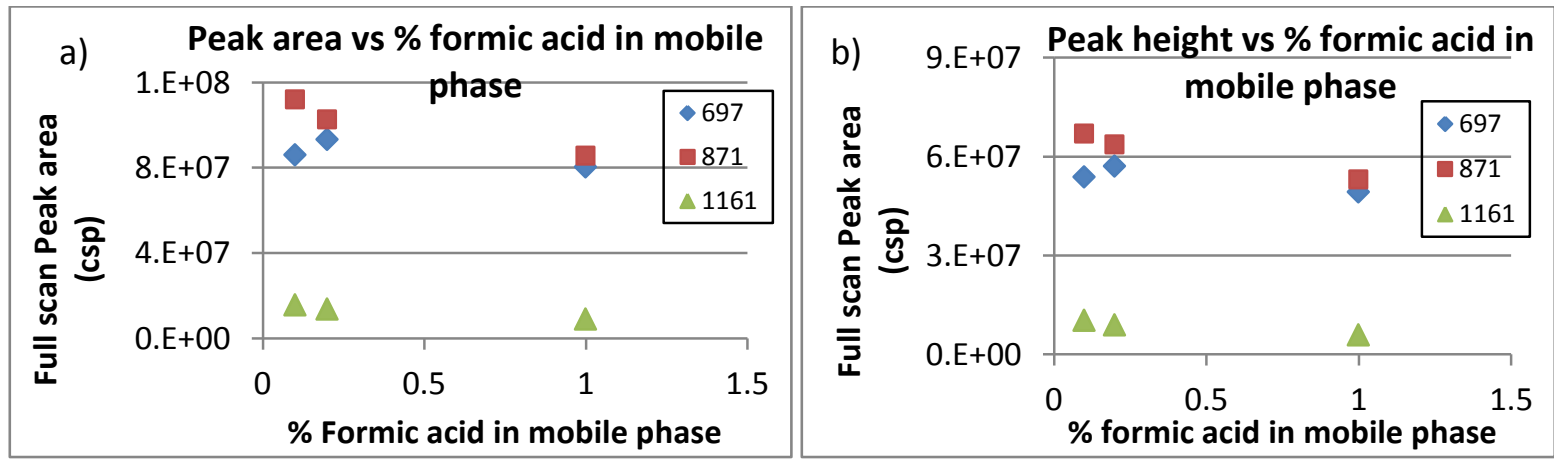

Figure S4 Effect of the formic acid content on glucagon (a) peak area and (b) height. Flow rate is $0.8 \mathrm{~mL} / \mathrm{mL}$, mobile phases are $A=A C N$ and $B=$ water, each modified with formic acid to the extent shown in the figure.

\subsection{Column clean time}

The original LC method included a 4 minute column clean step at the end of each injection at the LC starting conditions (22\% organic). This was found necessary to reduce column fouling, and resulted in a run time of 7.1 minutes. To attempt to reduce run time this was reduced to 0.4 minutes, which as expected led to column fouling during the injection of samples extracted using the original extraction method (Figure S5a vs b), however peak 

shape was maintained using the re-optimised extraction method with automated liquid transfer (Figure S5c vs d). This was attributed to the reduction of the inadvertent transfer of protein precipitate solid, and subsequent reduction of matrix build up on the column. The total run time for the final multiplexed method was 3.6 minutes, saving 5 hours of analysis time per 96 sample batch.
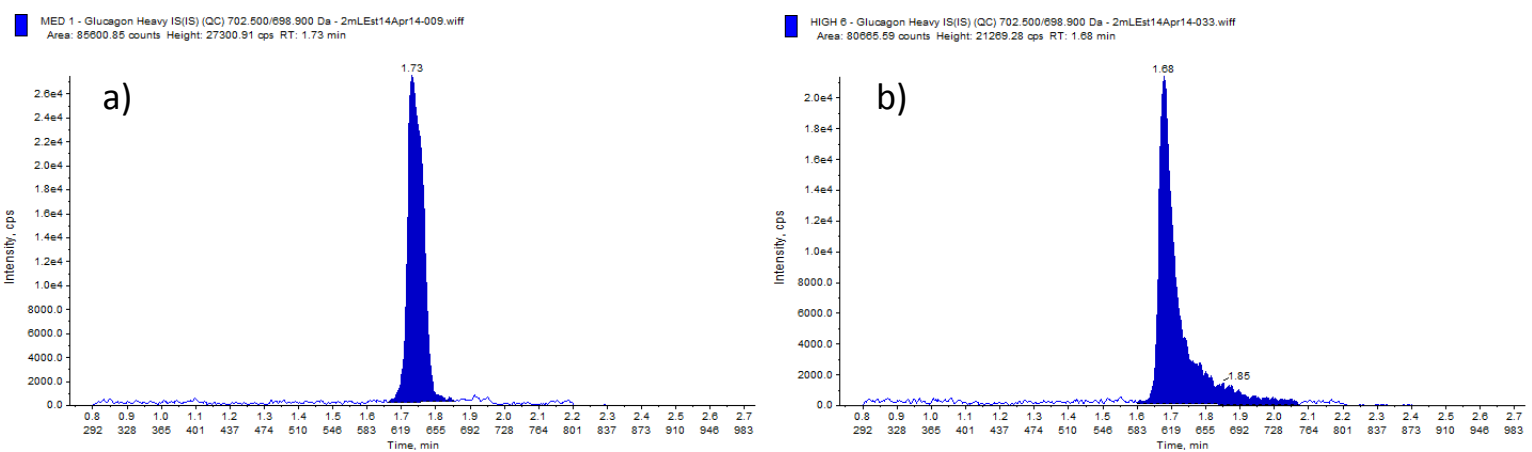

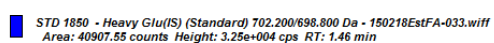

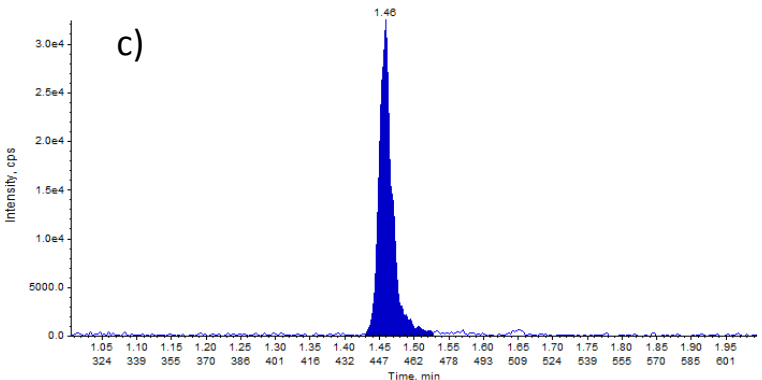

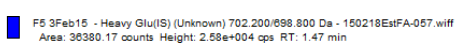

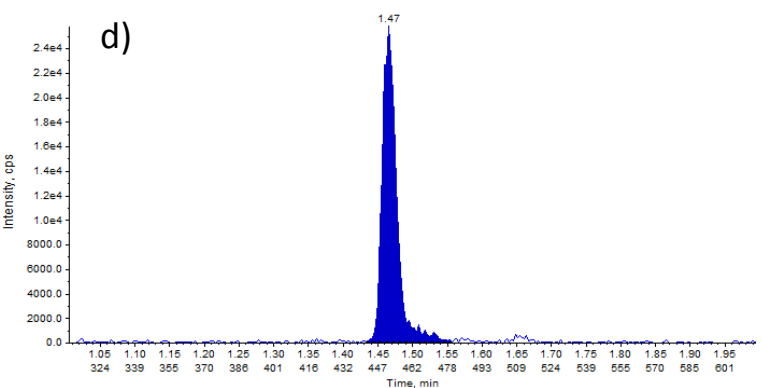

Figure S5 Comparison of SIL internal standard peak shape using the original extraction procedure at plasma injection a) 9 and b) 33, and using the re-optimised extraction procedure at plasma injection a) 9 and b) 33 .

\section{Internal Standard}

Table 3 Performance of the analogue and SIL glucagon internal standard against plasma QC samples extracted using the original [1] or re-optimised extraction procedure with plasma samples

\begin{tabular}{|c|c|c|c|c|c|}
\hline \multirow{2}{*}{$\begin{array}{c}\text { Plasma QC } \\
\text { Concentration }\end{array}$} & \multirow{2}{*}{$\begin{array}{c}\text { Extraction } \\
(\mathrm{pg} / \mathrm{mL})\end{array}$} & \multicolumn{4}{|c|}{ Glucagon Internal Standard } \\
\cline { 3 - 6 } & & \multicolumn{2}{|c|}{ Anologue } & \multicolumn{2}{|c|}{ SIL } \\
\cline { 3 - 6 } & & $\%$ Accuracy & \%CV & \% Accuracy & $\% \mathrm{CV}$ \\
\hline $\mathrm{MED}$ & Original & 114.0 & 6.2 & 97.8 & 4.3 \\
\cline { 3 - 6 } $100 \mathrm{pg} / \mathrm{mL}$ & Re-optimised & 98.5 & 5.5 & 92.0 & 5.5 \\
\hline $\mathrm{HIGH}$ & Original & 114.8 & 2.9 & 97.6 & 3.6 \\
$750 \mathrm{pg} / \mathrm{mL}$ & Re-optimised & 102.9 & 3.5 & 100.4 & 4.5 \\
\hline
\end{tabular}




\section{Mobile Phase Supercharging Reagents}

\subsection{Initial Optimisation}

\subsubsection{Optimisation of $m$-NBA content in mobile phases}

107 The percentage of $m$-NBA in the mobile phases was found to have a large effect on the charge state distribution of glucagon (Figure S6). As expected higher percentages favoured the most highly charged species $(6+, m / z=581)$. At low levels of $m$-NBA $(0-0.50 \%)$ the response of both the $(6+, m / z=581)$ and $6+(m / z=697)$ ions increased with $m$-NBA concentration, presumably due to an increase in overall ionisation efficiency. Whereas at higher levels of $m$-NBA the increase of the $6+(m / z=581)$ charge state was at the expense of the others. Data points were only collected for the $3+$ and $4+$ ions at 0 and $0.1 \% m-N B A$ concentrations, as these ions were least intense and therefore discarded early on in method development.

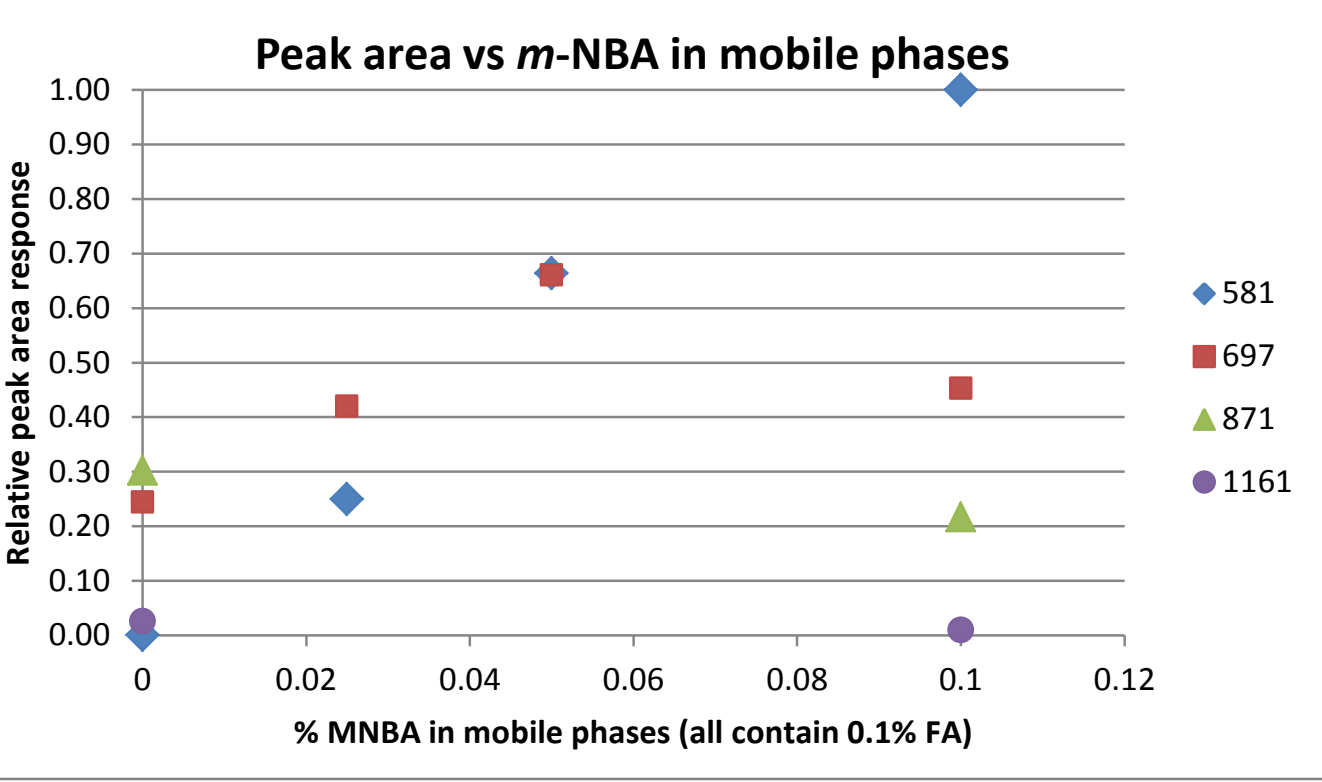

Figure S6 The effect of $m$-NBA mobile phase content on charge state distribution. Mobile phases are $\mathrm{A}=\mathrm{ACN}, \mathrm{B}=\mathrm{H}_{2} \mathrm{O} / \mathrm{ACN}(95 / 5)$, both modified with $m-\mathrm{NBA}$ as shown. $\mathrm{A} 1000 \mathrm{pg} / \mathrm{mL}$ surrogate standard ( $n=3$ replicates) was analysed in each case. These data are from 3 different experiments (Exp1 (full scan): 0 \& 0.1\% m-NBA, Exp2 (SRM): $0.025 \& 0.050 \% \mathrm{~m}$-NBA, Exp 3 (SRM):0.05 \& 0.1\% m-NBA). SRM transitions were 697/693 and 581/575. Data has been scaled appropriately, using common conditions between experiments, to account for signal variations. 


\subsubsection{Optimisation of Formic acid content in mobile phases}

The effect of adjusting the percentage of formic acid in mobile phases was relatively small in standard mobile phases (Section 3.2), however when $m-N B A$ was present the effect was significant (Figure S7). Acids are known to decrease the surface tension of electrospray droplets[2], and therefore altering concentrations could affect the supercharging process, which relies on increases in surface tensions by $m$-NBA [2].
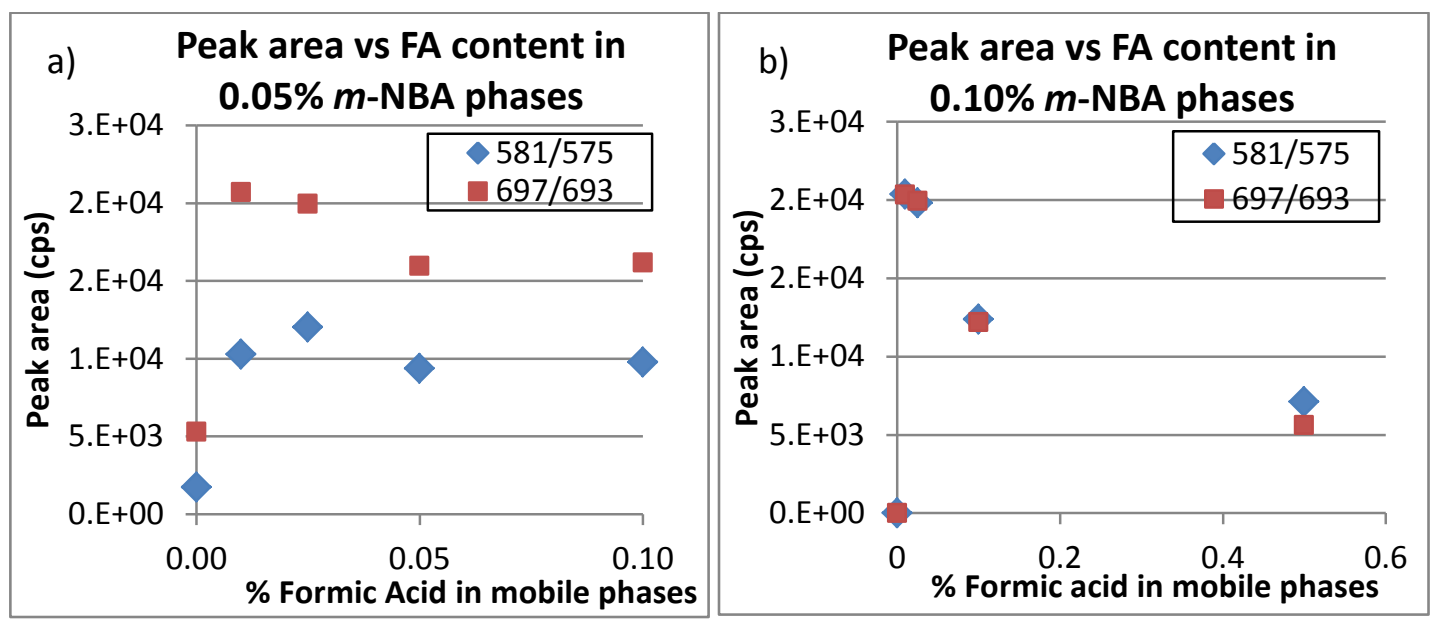

Figure 57 The effect of formic acid content on glucagon SRM peaks area using $\boldsymbol{m}$-NBA based mobile phases. Mobile phases are $\mathrm{A}=\mathrm{ACN}, \mathrm{B}=\mathrm{H}_{2} \mathrm{O} / \mathrm{ACN}(95 / 5)$, both modified with either (a) $0.05 \% \mathrm{~m}$-NBA with at $0,0.010,0.025,0.050$, or $0.0100 \% \mathrm{FA}$, or (b) $0.01 \% \mathrm{~m}$-NBA with at $0,0.010$, $0.025,0.0100$, or $0.500 \%$ FA. Values are means of $n \geq 3$ replicate injections of $1000 \mathrm{pg} / \mathrm{mL}$ surrogate matrix standards.

\subsection{Effect of $m$-NBA modified mobile phases upon analysis of GLP-1 and Glucagon Solutions and Plasma Extracts}

A SRM method was created with 13 transitions (8 GLP-1 transitions and 5 for Glucagon or Glucagon IS) (Figure S8 and Figure S9). The SRM transitions encompassed the 5+ and 6+ parent ions for glucagon, and the 3, 4, 5 and 6+ parent ions for GLP-1. Product ions were identified by fragmentation of these parent ions, followed by collision energy optimisation. Multiple transitions were monitored to increase the likelihood of finding those with low background noise (thereby increasing signal to noise) along with the absence of interferences. A greater number of GLP-1 transitions were selected, as glucagon SRM transitions had been previously investigated.

A $20 \mathrm{~ms}$ dwell time was used to ensure sufficient data points across the peaks. Scheduled MRM was used to maximise dwell time by only monitoring glucagon and GLP-1 transitions near their RT (1.6 and 2.5 respectively), a target scan time of 0.2 seconds, and MRM detection window of 30 seconds was selected.

Surrogate matrix and extracted plasma samples spiked to the $1200 \mathrm{pg} / \mathrm{mL}$ level with glucagon and GLP-1 were analysed using mobile phase modified with $0.05 \%$ or 
153 glucagon solutions were analysed (Section 5.1.1). The mobile phases also contained either $1540.01 \%$ FA, found to be optimal for glucagon solution analysis (Section 5.1 .2 ) or $0.1 \%$ FA. 155 The higher acid concentration was evaluated to investigate whether this was needed for 156 effective analyte ionisation due to competition from matrix components.

157 The effect of altering mobile phases was similar for surrogate matrix (solution) samples and 158 plasma extracts for both glucagon (Figure S8) and GLP-1 (Figure S9). In most cases it was 159 noted that plasma extracts gave a lower signal, although this will at least in part be due to 160 the loss of compound during the extraction, rather than difference in ionisation between the 161 matrices. Mobile phases containing $0.05 \%$ and $m$-NBA $0.01 \%$ FA were found to give 162 maximum signal, regardless of transition, nature of sample (plasma or solution), and analyte. The presence of matrix therefore did not alter the supercharging process. It was noted that some transitions were affected by the alteration in mobile phases more than others; demonstrating that supercharging of the analyte alters the intensity of various product ions formed.

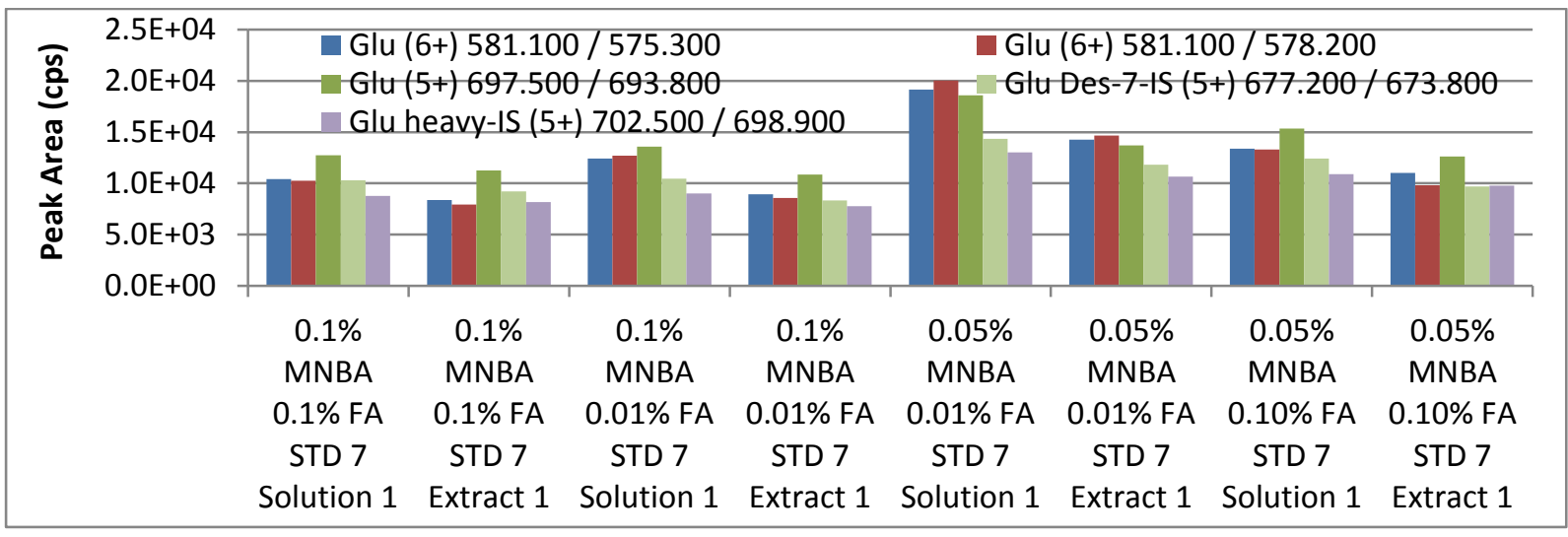

Figure S8 Glucagon peak areas for $1200 \mathrm{pg} / \mathrm{mL}$ solution and plasma extracts under different mobile phase conditions. $1200 \mathrm{pg} / \mathrm{mL}$ solution or plasma extracts were analysed, values are means of $n=3$ replicates.

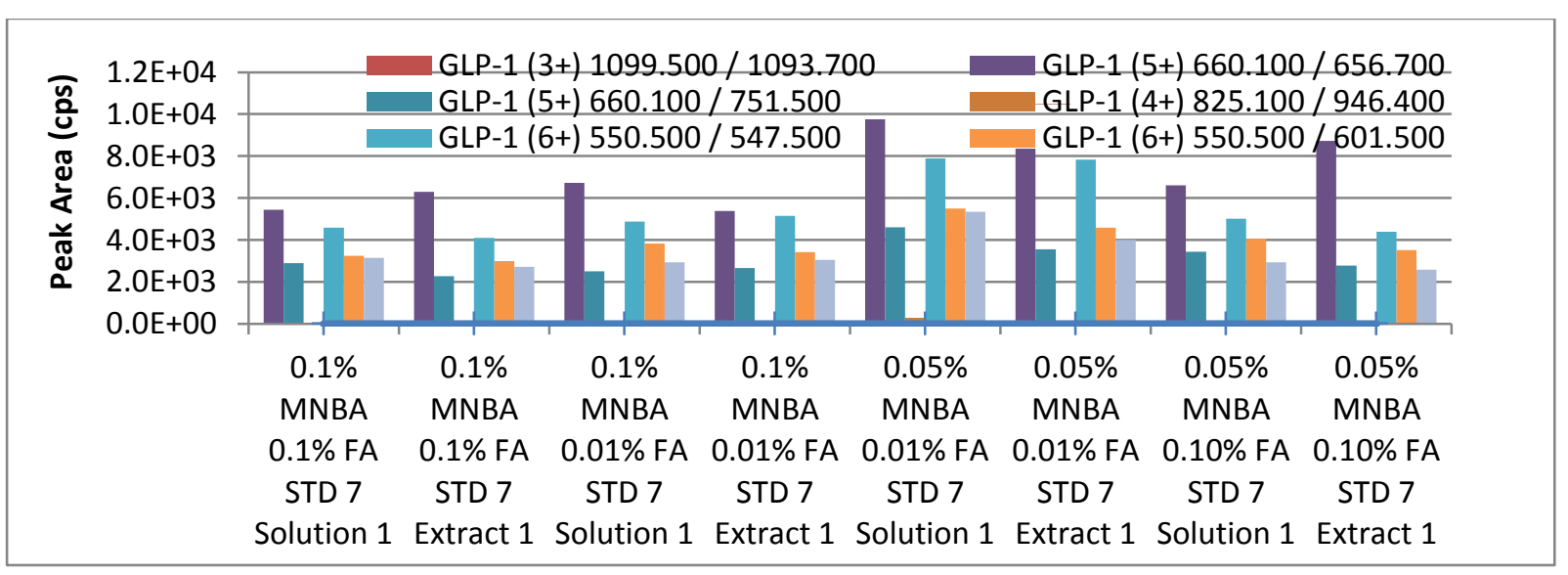


Figure S9 GLP-1 peak areas for $1200 \mathrm{pg} / \mathrm{mL}$ solution and plasma extracts under different mobile phase conditions. $1200 \mathrm{pg} / \mathrm{mL}$ solution or plasma extracts were analysed, values are means of $n=3$ replicates.

\subsection{Comparisons of the optimised $m$-NBA modified mobile phase with the original} formic acid mobile phase method

A plasma sample was spiked with $1200 \mathrm{pg} / \mathrm{mL}$ glucagon and GLP-1, and analysed with optimal $m$-NBA based mobile phase method (modified with $0.05 \% \mathrm{~m}$-NBA $0.01 \% \mathrm{FA}$ ) and the original formic acid based method (modified with $0.2 \%$ FA). The signal-to-noise was assessed for each transition monitored (Table 4).

The transitions highlighted were selected for further assessments using either standard mobile phases (blue) or supercharging mobile phase (red). Although these were not always the transitions that gave the best $\mathrm{S} / \mathrm{N}$, they gave best overall performance when linearity and precision and accuracy were considered.

The SRM method was subsequently altered to remove redundant transitions, and split into separate methods for method for analysis with formic acid and supercharging mobile phase methods to maximise dwell time for each transitions to improve sensitivity. In addition scheduled MRM was replaced with periods, as this was found to improve signal.

Table $4 \quad$ Signal-to-noise in various SRMs obtained by analysis of a $1200 \mathrm{pg} / \mathrm{mL}$ glucagon and GLP1 plasma extract, analysed using $m$-NBA or the original formic acid modified mobile phases.

\begin{tabular}{|c|c|c|c|c|c|}
\hline \multirow{2}{*}{$\begin{array}{l}\text { Glucagon } \\
\text { transition }\end{array}$} & \multicolumn{2}{|c|}{$\mathrm{S} / \mathrm{N}$} & \multirow{2}{*}{$\begin{array}{l}\text { GLP-1 } \\
\text { transition }\end{array}$} & \multicolumn{2}{|c|}{$\mathrm{S} / \mathrm{N}$} \\
\hline & $0.2 \% \mathrm{FA}$ & $\begin{array}{l}0.05 \% \text { MNBA } \\
0.01 \% \text { FA }\end{array}$ & & $0.2 \% \mathrm{FA}$ & $\begin{array}{l}0.05 \% \text { MNBA } \\
0.01 \% \text { FA }\end{array}$ \\
\hline $581.1 / 575.3$ & $\mathrm{~N} / \mathrm{A}$ & 31.1 & $550.5 / 547.5$ & $\mathrm{~N} / \mathrm{A}$ & 18.0 \\
\hline $581.1 / 578.2$ & $\mathrm{~N} / \mathrm{A}$ & 26.9 & $550.5 / 601.5$ & $\mathrm{~N} / \mathrm{A}$ & 14.5 \\
\hline $697.5 / 639.8$ & 17.6 & 15.4 & $550.5 / 639.3$ & $\mathrm{~N} / \mathrm{A}$ & 10.8 \\
\hline & & & $660.1 / 656.7$ & 12.0 & 3.4 \\
\hline & & & $660.1 / 751.5$ & 7.0 & 8.1 \\
\hline & & & $825.1 / 458.2$ & $\mathrm{~N} / \mathrm{A}$ & $\mathrm{N} / \mathrm{A}$ \\
\hline & & & $825.1 / 946.4$ & 4.1 & $\mathrm{~N} / \mathrm{A}$ \\
\hline & & & $1099.5 / 1093.7$ & $\mathrm{~N} / \mathrm{A}$ & $\mathrm{N} / \mathrm{A}$ \\
\hline
\end{tabular}

Mobile phases are $A=A C N$ and $B=$ water (or water/ACN 95/5 for $m$-NBA modification), modified as shown in the table. N/A- not applicable, as no peak was observed 
5.4 Comparison of SIL internal standard response of a surrogate matrix and extracted plasma sample

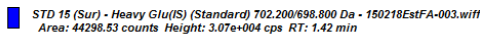

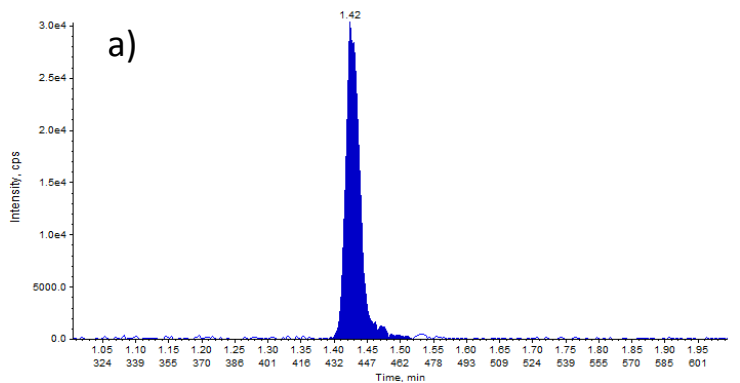

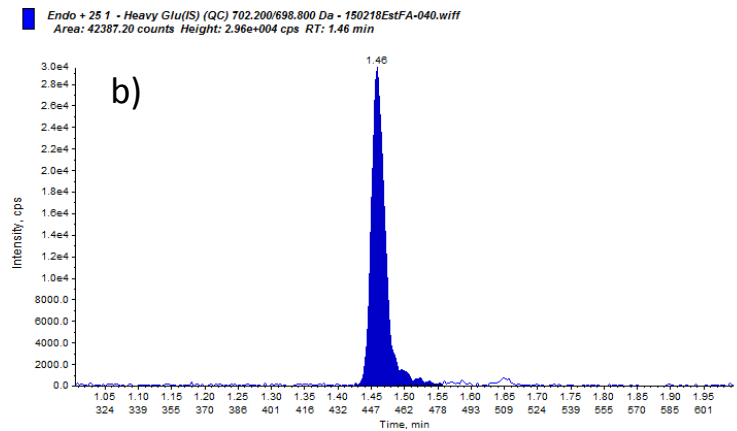

SIL gluagon internal standard response in a) a non-extracted surrogate matrix sample, and b) an extracted plasma sample 
6 Establishment Procedures (Supplemental Tables)

Table 5 Precision and accuracy of surrogate matrix and extracted plasma glucagon samples analysed using LC-MS/MS with either the original formic acid modified mobile phases, or $m$-NBA modified mobile phases

\begin{tabular}{|c|c|c|c|c|c|c|}
\hline \multirow[t]{2}{*}{ Sample ID } & \multirow[t]{2}{*}{$\begin{array}{l}\text { Surrogate/ } \\
\text { Plasma }\end{array}$} & \multirow[t]{2}{*}{$\begin{array}{c}\text { Nominal } \\
\text { concentration } \\
(\mathrm{pg} / \mathrm{mL})\end{array}$} & \multicolumn{2}{|c|}{$\begin{array}{c}\text { 0.2\% FA Phases } \\
\text { Glucagon } \\
(697.5 / 694.0)\end{array}$} & \multicolumn{2}{|c|}{$\begin{array}{c}0.05 \% m-\mathrm{NBA} \\
0.01 \% \text { FA phases } \\
\text { Glucagon } \\
(581.5 / 578.5)\end{array}$} \\
\hline & & & \%RE & $\% \mathrm{CV}$ & \%RE & $\% \mathrm{CV}$ \\
\hline QC LLOQ & Surrogate & 15 & 110.3 & 11.9 & 96.9 & 15.4 \\
\hline QC LOW & Surrogate & 45 & 113.8 & 6.2 & 110.9 & 11.8 \\
\hline$\left(\mathrm{QC}\right.$ LLOQ) ${ }^{*}$ & Plasma & $25^{1}$ & 92.6 & 7.0 & 90.6 & 10.0 \\
\hline$(Q C \text { LOW })^{*}$ & Plasma & $75^{1}$ & 92.4 & 11.3 & 99.6 & 10.9 \\
\hline QC MED & Plasma & $200^{1}$ & 91.4 & 8.7 & 100.0 & 9.7 \\
\hline QC MED (Lith Hep) & Plasma & $200^{2}$ & 92.6 & 9.0 & 119.0 & 9.3 \\
\hline QC MED (inter matrix) & Plasma & $200^{3}$ & 106.3 & 4.5 & 111.7 & 5.3 \\
\hline QC Dilution & Plasma & $1750^{4}$ & 84.9 & 6.7 & 78.1 & 8.3 \\
\hline $\mathrm{QC} \mathrm{HIGH}$ & Plasma & $1750^{1}$ & 98.0 & 10.7 & 106.1 & 12.2 \\
\hline
\end{tabular}

206

207

Green statistics are within $20 \%$ RE or $<20 \%$ CV for glucagon, or within $25 \%$ RE or $<25 \%$ CV for GLP-1

* = QC level created for GLP-1 analysis, but additionally monitored for glucagon to improve assay characterisation

$1=31.9 \mathrm{pg} / \mathrm{mL} / 37.5 \mathrm{pg} / \mathrm{mL}$ endogenous glucagon measured using formic acid / m-NBA phases, and added to the spiked concentration

$2=$ No endogenous glucagon detected in lithium heparin plasma used

$3=$ Adjusted for endogenous glucagon concentration determined in each individual matrix $4=$ No endogenous glucagon detected in matrix used for dilution QCs 
Table 6 Glucagon matrix effects determined using LC-MS/MS with either the original formic acid modified mobile phases, or $\boldsymbol{m}$-NBA modified mobile phases

\begin{tabular}{|c|c|c|c|c|c|c|}
\hline $\begin{array}{c}\text { Matrix } \\
\text { ID }\end{array}$ & \multicolumn{3}{|c|}{$\begin{array}{c}0.2 \% \text { FA Phases } \\
\text { Glucagon (697.5/694.0) }\end{array}$} & \multicolumn{3}{c|}{$\begin{array}{c}0.05 \% \\
0.01 \% \text { FA phases } \\
\text { Glucagon (581.5/578.5) }\end{array}$} \\
\cline { 2 - 7 } & $\begin{array}{c}\text { Analyte } \\
\text { ME }\end{array}$ & $\begin{array}{c}\text { IS } \\
\text { ME }\end{array}$ & $\begin{array}{c}\text { ME } \\
\text { Ratio }\end{array}$ & $\begin{array}{c}\text { Analyte } \\
\text { ME }\end{array}$ & $\begin{array}{c}\text { IS } \\
\text { ME }\end{array}$ & $\begin{array}{c}\text { ME } \\
\text { Ratio }\end{array}$ \\
\hline F1 & 1.58 & 1.48 & 1.07 & 1.09 & 0.985 & 1.10 \\
\hline F2 & 1.36 & 1.43 & 0.951 & 1.01 & 1.05 & 0.961 \\
\hline F4 & 1.33 & 1.34 & 0.992 & 0.924 & 0.899 & 1.03 \\
\hline F5 & 1.30 & 1.47 & 0.882 & 1.16 & 1.19 & 0.979 \\
\hline M1 & 1.33 & 1.21 & 1.10 & 0.922 & 0.792 & 1.16 \\
\hline M4 & 1.32 & 1.20 & 1.09 & 0.656 & 0.591 & 1.11 \\
\hline Mean & 1.37 & 1.36 & 1.01 & 0.960 & 0.918 & 1.06 \\
\hline SD & 0.104 & 0.127 & 0.0881 & 0.176 & 0.209 & 0.0806 \\
\hline \%CV & 7.615 & 9.34 & 8.7 & 18.354 & 22.82 & 7.62 \\
\hline
\end{tabular}

Adjusted Area $=$ Peak area of plasma ME sample- Peak area of endogenous plasma sample 
Table 7 Precision and accuracy of extracted plasma GLP-1 samples analysed using LC-MS/MS with formic acid (top) or $m$-NBA (bottom) modified mobile phases with either the analogue or SIL glucagon internal standard. Green statistics are within $25 \% \mathrm{RE}$ or $<25 \% \mathrm{CV}$. Red statistics are outside these criteria. NA -not analysed, as $660.1 / 751.5$ was determined to be optimal before these experiments were conducted.

\begin{tabular}{|c|c|c|c|c|c|c|c|c|c|c|c|c|c|c|}
\hline \multirow[t]{3}{*}{ Sample ID } & \multirow[t]{3}{*}{$\begin{array}{l}\text { Surrogate/ } \\
\text { Plasma }\end{array}$} & \multirow{3}{*}{$\begin{array}{c}\text { Nominal } \\
\text { concentration } \\
(\mathrm{pg} / \mathrm{mL})\end{array}$} & \multicolumn{6}{|c|}{$\begin{array}{l}0.2 \% \text { FA Phases } \\
\text { GLP-1 (660.1/656.7) }\end{array}$} & \multicolumn{6}{|c|}{$\begin{array}{l}0.2 \% \text { FA Phases } \\
\text { GLP-1 (660.1/751.5) }\end{array}$} \\
\hline & & & \multicolumn{2}{|c|}{ No IS } & \multicolumn{2}{|c|}{$\begin{array}{c}\text { Heavy } \\
\text { Glucagon }\end{array}$} & \multicolumn{2}{|c|}{$\begin{array}{l}\text { Des-7-Thr- } \\
\text { Glucagon }\end{array}$} & \multicolumn{2}{|c|}{ No IS } & \multicolumn{2}{|c|}{$\begin{array}{c}\text { Heavy } \\
\text { Glucagon }\end{array}$} & \multicolumn{2}{|c|}{$\begin{array}{c}\text { Des-7-Thr- } \\
\text { Glucagon }\end{array}$} \\
\hline & & & $\% R E$ & $\% \mathrm{CV}$ & $\% R E$ & $\% \mathrm{CV}$ & $\% R E$ & $\% \mathrm{CV}$ & $\% R E$ & $\% \mathrm{CV}$ & $\% R E$ & $\% \mathrm{CV}$ & $\% R E$ & $\% \mathrm{CV}$ \\
\hline QC LLOQ & Plasma & 25 & 102.2 & 18.2 & 88.0 & 11.6 & 86.9 & 16.7 & 131.4 & 20.1 & 118.6 & 20.0 & 112.7 & 24.6 \\
\hline QC LOW & Plasma & 75 & 102.6 & 13.1 & 113.1 & 13.6 & 113.8 & 11.7 & 103.7 & 22.4 & 110.2 & 11.3 & 107.4 & 13.6 \\
\hline QC MED & Plasma & 200 & 107.9 & 16.6 & 107.4 & 7.7 & 109.6 & 8.4 & 108.6 & 10.7 & 107.3 & 2.0 & 107.2 & 6.7 \\
\hline QC MED (Lith Hep) & Plasma & 200 & NA & NA & NA & NA & NA & NA & 72.9 & 15.6 & 81.9 & 12.6 & 75.2 & 12.9 \\
\hline QC MED (inter matrix) & Plasma & 200 & NA & NA & NA & NA & NA & NA & 149.1 & 15.4 & 134.5 & 16.0 & 122.9 & 16.3 \\
\hline QC DIL & Plasma & 10,000 & 73.1 & 9.6 & 72.1 & 10.5 & 74.7 & 11.8 & 76.6 & 6.7 & 75.7 & 8.8 & 76.8 & 10.0 \\
\hline QC HIGH & Plasma & 1750 & 99.7 & 15.6 & 111.6 & 2.7 & 113.9 & 5.8 & 102.9 & 15.5 & 113.8 & 3.6 & 113.8 & 6.7 \\
\hline
\end{tabular}

\begin{tabular}{|c|c|c|c|c|c|c|c|c|c|c|c|c|c|c|}
\hline \multirow[t]{3}{*}{ Sample ID } & \multirow[t]{3}{*}{$\begin{array}{c}\text { Surrogate/ } \\
\text { Plasma }\end{array}$} & \multirow{3}{*}{$\begin{array}{c}\text { Nominal } \\
\text { concentration } \\
(\mathrm{pg} / \mathrm{mL})\end{array}$} & \multicolumn{6}{|c|}{$\begin{array}{c}0.05 \% \text { m-NBA } 0.01 \% \text { FA phases } \\
(550.5 / 601.5)\end{array}$} & \multicolumn{6}{|c|}{$\begin{array}{c}0.05 \% \text { m-NBA } 0.01 \% \text { FA phases } \\
(550.5 / 693.3)\end{array}$} \\
\hline & & & \multicolumn{2}{|c|}{ No IS } & \multicolumn{2}{|c|}{$\begin{array}{c}\text { Heavy } \\
\text { Glucagon }\end{array}$} & \multicolumn{2}{|c|}{$\begin{array}{l}\text { Des-7-Thr- } \\
\text { Glucagon }\end{array}$} & \multicolumn{2}{|c|}{ No IS } & \multicolumn{2}{|c|}{$\begin{array}{c}\text { Heavy } \\
\text { Glucagon }\end{array}$} & \multicolumn{2}{|c|}{$\begin{array}{l}\text { Des-7-Thr- } \\
\text { Glucagon }\end{array}$} \\
\hline & & & $\%$ RE & $\% \mathrm{CV}$ & $\% \mathrm{RE}$ & $\% \mathrm{CV}$ & $\%$ RE & $\% \mathrm{CV}$ & $\%$ RE & $\% \mathrm{CV}$ & $\% \mathrm{RE}$ & $\% \mathrm{CV}$ & $\% \mathrm{RE}$ & $\% \mathrm{CV}$ \\
\hline QC LLOQ & Plasma & 25 & 88.0 & 25.6 & 75.1 & 36.6 & 75.1 & 33.8 & 75.0 & 43.2 & 57.5 & 39.8 & 63.4 & 33.0 \\
\hline QC LOW & Plasma & 75 & 83.9 & 17.3 & 107.0 & 19.6 & 103.9 & 15.8 & 71.0 & 15.5 & 104.4 & 25.3 & 96.0 & 22.8 \\
\hline QC MED & Plasma & 200 & 80.2 & 14.1 & 94.8 & 8.8 & 94.4 & 7.2 & 75.0 & 13.6 & 91.4 & 11.5 & 88.3 & 12.8 \\
\hline QC MED (Lith Hep) & Plasma & 200 & NA & NA & NA & NA & 59.9 & 19.4 & NA & NA & NA & NA & NA & NA \\
\hline QC MED (inter matrix) & Plasma & 200 & NA & NA & NA & NA & 177.1 & 37.5 & NA & NA & NA & NA & NA & NA \\
\hline QC DIL & Plasma & 10,000 & 63.8 & 12.4 & 75.8 & 7.4 & 74.6 & 8.3 & 63.6 & 13.7 & 83.1 & 7.2 & 76.6 & 7.9 \\
\hline QC HIGH & Plasma & 1750 & 80.1 & 12.8 & 99.6 & 16.8 & 107.4 & 12.2 & 78.7 & 13.6 & 103.2 & 18.4 & 106.8 & 14.1 \\
\hline
\end{tabular}


Table 8 GLP-1 matrix effects determined using LC-MS/MS with the original formic acid modified mobile phase and the analogue glucagon internal standard. See footnotes on Table 6.

\begin{tabular}{|c|c|c|c|}
\hline \multirow{2}{*}{ Matrix ID } & \multicolumn{3}{|c|}{$0.2 \%$ FA Phases } \\
& GLP-1 (660.1/751.5) with analogue glucagon IS \\
\cline { 2 - 4 } & Analyte ME & IS ME & Ratio \\
\hline F1 & 1.14 & 1.41 & 0.808 \\
\hline F2 & 0.869 & 1.33 & 0.653 \\
\hline F4 & 0.876 & 1.40 & 0.628 \\
\hline F5 & 0.735 & 1.34 & 0.548 \\
\hline M1 & 1.02 & 1.24 & 0.827 \\
\hline M4 & 0.765 & 1.19 & 0.641 \\
\hline Mean & 0.901 & 1.32 & 0.684 \\
\hline SD & 0.154 & 0.0860 & 0.110 \\
\hline \%CV & 17.1 & 6.5 & 16.1 \\
\hline
\end{tabular}

Table 9 Inter-batch reproducibility of endogenous glucagon determination.

\begin{tabular}{|c|c|c|}
\hline \multirow{2}{*}{ Replicate } & \multicolumn{2}{|c|}{ Measured Endogenous Glucagon Concentration $(\mathrm{pg} / \mathrm{mL})$} \\
\cline { 2 - 3 } & Initial Analysis & $\begin{array}{c}\text { Reanalysis after 42 Days } \\
\text { storage at }-80^{\circ} \mathrm{C}\end{array}$ \\
\hline 1 & 29.7 & 34.0 \\
\hline 2 & 31.1 & 36.5 \\
\hline 3 & 33.8 & 31.7 \\
\hline 4 & 32.7 & 35.7 \\
\hline 5 & 34.2 & 33.8 \\
\hline 6 & 29.9 & 27.9 \\
\hline Mean & 31.9 & 33.3 \\
\hline SD & 1.95 & 3.11 \\
\hline CV & 6.1 & 9.4 \\
\hline \% Difference & \multicolumn{2}{|c}{} \\
\hline
\end{tabular}


Table 10 Performance of QCs in stabilised plasma vs non-stabilised plasma.

\begin{tabular}{|c|c|c|c|c|}
\hline Anticoagulant & Stabiliser & $\begin{array}{c}\text { Freeze-thaw } \\
\text { cycles }\end{array}$ & \multicolumn{2}{|c|}{$\begin{array}{c}\text { Non-stabilised } \\
\text { QC concentration }\end{array}$} \\
\cline { 4 - 5 } & & & Glucagon & GLP-1 \\
\hline EDTA & None & 0 & 113.3 & 77.9 \\
\hline EDTA & & 0 & 94.8 & 99.7 \\
\hline EDTA & $\mathrm{D}$ & 0 & 102.9 & 104.9 \\
\hline EDTA & AD & 0 & 109.1 & 107.0 \\
\hline EDTA & AD & 1 & 101.1 & 86.2 \\
\hline EDTA & AD & 2 & 109.5 & 95.0 \\
\hline Lithium Heparin & None & 0 & 81.7 & 84.1 \\
\hline Lithium Heparin & $\mathrm{A}$ & 0 & 98.1 & 97.1 \\
\hline Lithium Heparin & $\mathrm{D}$ & 0 & 91.1 & 105.9 \\
\hline Lithium Heparin & $\mathrm{AD}$ & 0 & 98.0 & 113.7 \\
\hline
\end{tabular}

Formic acid modified mobile phases used

$200 \mathrm{pg} / \mathrm{mL}$ of glucagon/GLP-1 was spiked into a variety of matrices, as shown, and compared to the performance of QCs spike in non-stabilised EDTA plasma.

Table $11 \quad$ Ability to reinject sample extracts after 42 days storage at $4^{\circ} \mathrm{C}$

\begin{tabular}{|c|c|c|c|c|c|}
\hline Surrogate/ & Nominal & \multicolumn{2}{|c|}{ Glucagon } & \multicolumn{2}{c|}{ GLP-1 } \\
Plasma Matrix & $\begin{array}{c}\text { Concentration } \\
(\mathrm{pg} / \mathrm{mL})\end{array}$ & $\% \mathrm{RE}$ & $\% \mathrm{CV}$ & $\% \mathrm{RE}$ & $\% \mathrm{CV}$ \\
\cline { 3 - 6 } & 15 & 107.1 & 11.6 & $\mathrm{~N} / \mathrm{A}$ & $\mathrm{N} / \mathrm{A}$ \\
\hline Surrogate & 25 & 106.0 & 11.3 & $\mathrm{~N} / \mathrm{A}$ & $\mathrm{N} / \mathrm{A}$ \\
\hline Surrogate & 45 & 111.3 & 7.8 & $\mathrm{~N} / \mathrm{A}$ & $\mathrm{N} / \mathrm{A}$ \\
\hline Surrogate & $25 \#$ & 86.5 & 7.6 & 116.6 & 7.4 \\
\hline Plasma & $25 \#$ & 87.7 & 10.1 & 99.3 & 24.8 \\
\hline Plasma & $75 \#$ & 89.6 & 10.0 & 98.4 & 7.3 \\
\hline Plasma & $200 \#$ & 89.0 & 13.6 & 104.7 & 22.2 \\
\hline Plasma & $1750 \#$ & 99.0 &
\end{tabular}

Formic acid modified mobile phases used.

\#31.4 pg/mL endogenous concentration added to glucagon QC concentration Green statistics are within $20 \%$ RE or $<20 \%$ CV (Glucagon) or 25\% RE or $<25 \%$ CV (GLP-1). 

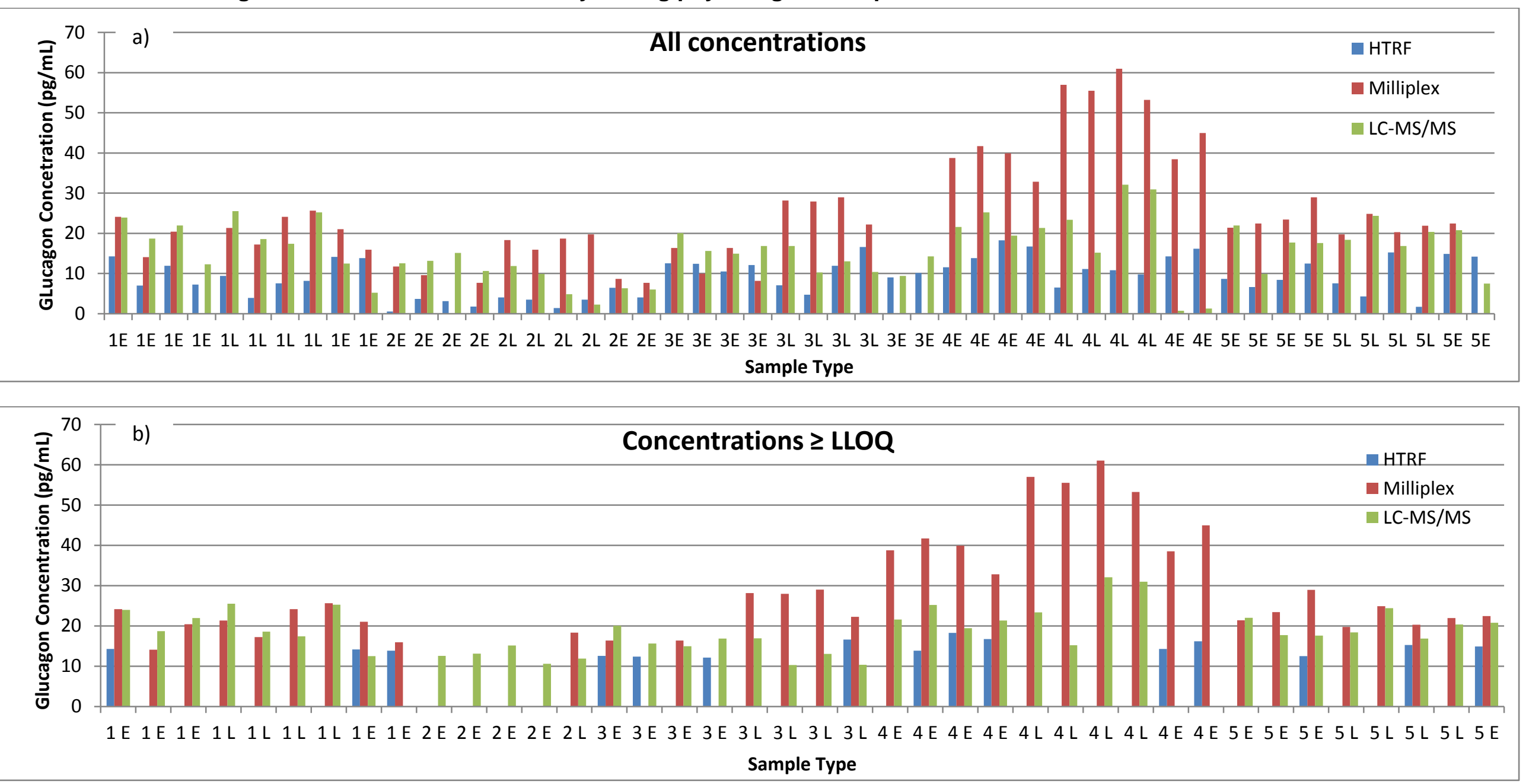

Figure S11 Comparison of endogenous glucagon concentrations determined by LC-MS/MS, Milliplex and HTRF immunoassays. Samples were analysed from 5 volunteers (1-5), which contained either EDTA (E) or Lithium Heparin (L) anticoagulant in addition to various stabilisers (not shown). Different stabiliser combinations were used in samples of the same Sample Type, e.g. the four 4E samples in Figure 11a are all unique. a) All concentrations determined. b) Concentrations above the LLOQ only shown ( $\geq 10.0 / 13.0$ / 12.0 pg/mL for LC-MS/MS / Milliplex / HTRF respectively). 


\section{References}

255

[1] Howard JW, Kay RG, Tan T, et al. Development of a high throughput UHPLC-MS/MS (SRM) method for the quantitation of endogenous glucagon from human plasma. Bioanalysis. 6(24), 3295-3309 (2014).

[2] lavarone AT, Williams ER. Mechanism of charging and supercharging molecules in electrospray ionization. J. Am. Chem. Soc. 125(8), 2319-27 (2003). 